\title{
SPARSE TENSOR PRODUCT WAVELET APPROXIMATION OF SINGULAR FUNCTIONS
}

\author{
MONIQUE DAUGE AND ROB STEVENSON
}

\begin{abstract}
On product domains, sparse-grid approximation yields optimal, dimension independent convergence rates when the function that is approximated has $L^{2}$-bounded mixed derivatives of a sufficiently high order. We show that the solution of Poisson's equation on the $n$-dimensional hypercube with Dirichlet boundary conditions and smooth right-hand side generally does not satisfy this condition. As suggested by P.-A. Nitsche in [Constr. Approx., 21(1) (2005), pp. 63-81], the regularity conditions can be relaxed to corresponding ones in weighted $L^{2}$ spaces when the sparse-grid approach is combined with local refinement of the set of onedimensional wavelets indices towards the end points. In this paper, we prove that for general smooth right-hand sides, the solution of Poisson's problem satisfies these relaxed regularity conditions in any space dimension. Furthermore, since we remove log-factors from the energy-error estimates from Nitsche's work, we show that in any space dimension, locally refined sparse-grid approximation yields the optimal, dimension independent convergence rate.
\end{abstract}

\section{INTRODUCTION}

For standard, isotropic piecewise polynomial approximation of order $d$ on an $n$ dimensional domain $\Omega \subset \mathbb{R}^{n}$, it is well-known that the error, measured in $H^{m}(\Omega)$, in the best approximation of a sufficiently smooth function $u$ is generally of order $N^{-\frac{d-m}{n}}$, where $N$ denotes the dimension of the approximation space. The fact that the rate $\frac{d-m}{n}$ is inversely proportional to $n$ is known as the curse of dimensionality. When working on a product domain, this curse can be overcome by considering sparsegrid approximation ([Zen91, BG04]), also known as hyperbolic wavelet approximation ([DKT98]). With this type of approximation and $m \geq 0$, the error is of order $N^{-(d-m)}$, up to some log-factors, that for $m>0$ can even be removed by considering optimized sparse grids ([GK00]).

At this point we note that, in this paper, with an error of order $N^{-s}$, we mean that the error can be bounded by a multiple of $N^{-s}$, where this multiple is independent of $N$, but may depend on the space dimension $n$. Because of the latter, having shown that a convergence rate $s$ is independent of $n$ does not mean that the problem is tractable, that is, that the work for obtaining an error less than some tolerance $\varepsilon$ may not grow exponentially in $n$. We refer to [NW08, Wer96] and the references

Date: June 25, 2010.

2000 Mathematics Subject Classification. 35B65, 35J25, 41A25, 41A63, 42C40, 65N99, 65T60.

Key words and phrases. Wavelets, sparse grids, weighted anisotropic Sobolev spaces, regularity estimates, tensor products, local refinements. 
cited there for results dealing with (in)tractability of approximations in high space dimensions.

A sparse grid is based on a tensorized one-dimensional multiscale decomposition. In the literature, often hierarchical bases are used, also known as Faber bases. In this paper, we restrict ourselves to "true" one-dimensional wavelet decompositions, in the sense that, properly scaled, they will generate Riesz bases for a range of Sobolev spaces including $L^{2}$.

For having the aforementioned error bounds, it is needed that certain mixed partial derivatives of $u$ are bounded. For the model problem of $u$ being the solution of Poisson's problem on $\square=(0,1)^{n}$ with homogeneous Dirichlet boundary conditions, we will show that generally these regularity conditions are not fulfilled, regardless of the smoothness of the right-hand side $f$. Instead, we show that for sufficiently smooth $f$, generally the error in $H^{1}(\square)$ is only of order $N^{-\left(\frac{1}{2}+\frac{1}{n}\right)}$ (and also that the error is of order $N^{-(d-1)}$ when additionally $f$ vanishes at the order $d-1$ at the boundary).

Interior regularity theory shows that on any $\hat{\Omega} \subset \subset \square$ (as well as on the smooth parts of the boundary), any partial derivative of $u$ is bounded assuming a sufficient smoothness of $f$. So lacking smoothness of $u$ is caused by boundary singularities, more accurately, by edge and corner singularities. This fact motivates Nitsche in the pioneering work [Nit05] to combine sparse-grid approximation with local refinement of the underlying one-dimensional wavelet collection towards $\{0,1\}$. He showed that with this type of approximation, a function $u$ can be approximated in $H^{1}(\square)$-norm with an error of order $(\log N)^{t} N^{-(d-1)}$, for some $t>0$ depending on $n$ and $d$, assuming $u$ has mixed derivatives of sufficiently high order that are bounded in a weighted $L^{2}(\square)$-norm, with the weight being a product of one-dimensional smooth weights that vanish at $\{0,1\}$. Moreover, for the space dimension $n=2$, he showed that this regularity assumption is fulfilled for the solution of Poisson's problem on $\square$ with homogeneous Dirichlet boundary conditions when the right-hand side is sufficiently smooth (but does not necessarily vanish at the boundary).

In the present paper, we provide a new regularity result of the solution of Poisson's problem on $\square$ with homogeneous Dirichlet boundary conditions in certain anisotropic weighted Sobolev spaces. This result shows that the regularity assumptions made by Nitsche are fulfilled in any space dimension. At this point, we stress that evaluating regularity in anisotropic weighted spaces is mandatory as soon as the space dimension is larger or equal to 3: The presence of edges generally prevents the sufficient regularity to hold in isotropic weighted spaces.

Secondly, we remove the log-factors from the error estimate in $H^{1}(\square)$, which factors arose from two different estimates in [Nit05]. That is, we demonstrate the best possible, dimension independent convergence rate $d-1$ in $H^{1}(\square)$. The corresponding error estimate in $L^{2}(\square)$ will still contain some log-factors.

Thirdly, in [Nit05], the approximation results were derived with wavelets that do not satisfy boundary conditions. Thinking of $u$ being the solution of an (elliptic) boundary value problem with homogeneous Dirichlet boundary conditions, there is no obvious way how such wavelets can actually be used for solving such a boundary value problem. For approximating a function in $H_{0}^{1}(\square)$, we will use wavelets that are 
in that space. This will require some technicalities dealing with tensor products of weighted Sobolev spaces that incorporate essential boundary conditions.

Finally, we reduce the amount of local refinement needed to approximate the same type of boundary singularities, which may result in a quantitative improvement.

Note that the rates we demonstrate from the spans of wavelet sets constructed using a priori local refinements towards the boundaries are obviously also realized by best $N$-term approximations from the wavelet basis. The rate of best $N$-term approximation in turn can be realized, in linear complexity, by adaptive wavelet methods, cf. [DSS08].

This paper is organized as follows: Sect. 2 deals with wavelet approximation with local refinement of functions on the interval that are contained in certain weighted Sobolev spaces with a weight that vanishes at the end points.

In Sect. 3, we discuss tensor products of Hilbert spaces. In particular, we show that (weighted) Sobolev spaces on product domains that incorporate essential boundary conditions can be written as intersections of tensor products of such spaces in the coordinate directions.

In Sect. 4, we demonstrate optimal convergence rates with tensor product locally refined wavelet approximation of functions on $\square$ that are contained in (intersections of) tensor products of weighted Sobolev spaces.

Assuming a sufficiently smooth right-hand side, in Sect. 5 it is shown that the solution of Poisson's problem on $\square$ with homogeneous Dirichlet boundary conditions has arbitrarily high regularity in a suitable scale of anisotropic weighted Sobolev spaces.

Finally, in Sect. 6, we show that the solution of this Poisson problem generally has limited smoothness in the scale of (unweighted) Sobolev spaces of dominating mixed derivatives that governs the rate of approximation of (optimized) sparse grids without local refinements.

In this paper, by $C \lesssim D$ we will mean that $C$ can be bounded by a constant multiple of $D$, which constant, unless stated otherwise, is independent of parameters which $C$ and $D$ may depend on, possibly with the exception of the space dimension $n$. Obviously, $C \gtrsim D$ is defined as $D \lesssim C$, and $C \approx D$ as $C \lesssim D$ and $C \gtrsim D$.

\section{WAVELET APPROXIMATION OF SINGULAR FUNCTIONS IN ONE DIMENSION USING LOCAL REFINEMENT}

For $k \in \mathbb{N}_{0}, \theta \geq 0$, and with

$$
\mathrm{I}:=(0,1)
$$

let the weighted Sobolev space $H_{\theta}^{k}(\mathrm{I})$ be defined as the space of all measurable functions $u$ for which the norm

$$
\|u\|_{H_{\theta}^{k}(\mathrm{I})}:=\left[\sum_{j=0}^{k} \int_{\mathrm{I}}\left|x^{\theta} u^{(j)}(x)\right|^{2} d x\right]^{\frac{1}{2}}
$$

is finite. The semi-norm $\left[\int_{\mathrm{I}}\left|x^{\theta} u^{(k)}(x)\right|^{2} d x\right]^{\frac{1}{2}}$ will be denoted as $|u|_{H_{\theta}^{k}(0,1)}$. 
Lemma 2.1 ([Nit05, Lemma 2]). For $q \in[1, \infty]$ and $\theta-\left(k-\frac{1}{2}\right)<\frac{1}{q}$, we have the following continuous embedding

$$
H_{\theta}^{k}(\mathrm{I}) \hookrightarrow L^{q}(\mathrm{I})
$$

Remark 2.2. Since $H_{\theta_{1}}^{k}(\mathrm{I}) \hookrightarrow H_{\theta_{2}}^{k}(\mathrm{I})$ when $\theta_{1} \leq \theta_{2}$, clearly the additional condition $\theta>k-\frac{1}{2}$ imposed in [Nit05] can be omitted.

Remark 2.3. Lemma 2.1 is sharp in the sense that if $\theta-\left(k-\frac{1}{2}\right)>\frac{1}{q}$, then $H_{\theta}^{k}(\mathrm{I}) \hookrightarrow$ $L^{q}(\mathrm{I})$. Indeed, for $\alpha \in\left(k-\frac{1}{2}-\theta,-\frac{1}{q}\right), x \mapsto x^{\alpha} \in H_{\theta}^{k}(\mathrm{I})$, whereas this function is not in $L^{q}(\mathrm{I})$.

Remark 2.4. In the situation of Lemma 2.1 and for $j \in \mathbb{N}_{0}$, obviously also $H_{\theta}^{k+j}(\mathrm{I}) \hookrightarrow$ $W^{j, q}(\mathrm{I})$.

For $k \in \mathbb{N}, q \in[1, \infty], \Omega$ being a domain in $\mathbb{R}^{n}$, and $\Gamma \subset \partial \Omega, W_{0, \Gamma}^{k, q}(\Omega)$ will denote the closure in $W^{k, q}(\Omega)$ of the space of smooth functions on $\Omega$ whose supports have empty intersection with the Dirichlet boundary $\Gamma$. As usual, $W^{k, 2}(\Omega)$ is denoted as $H^{k}(\Omega)$ and $W_{0, \Gamma}^{k, 2}(\Omega)$ as $H_{0, \Gamma}^{k}(\Omega)$.

For some $m \in \mathbb{N}$ and $z \subseteq \partial \mathrm{I}=\{0,1\}$, we assume a wavelet collection

$$
\left\{\psi_{\lambda}: \lambda \in \nabla\right\} \subset H_{0, z}^{m}(\mathrm{I})
$$

such that

(1) $\left\{\psi_{\lambda}: \lambda \in \nabla\right\}$ is a Riesz basis for $L^{2}(\mathrm{I})$,

(2) $\left\{2^{-|\lambda| m} \psi_{\lambda}: \lambda \in \nabla\right\}$ is a Riesz basis for $H_{0, z}^{m}(\mathrm{I})$,

where $|\lambda| \in \mathbb{N}_{0}$ denotes the level of $\psi_{\lambda}$ or that of $\lambda$. Denoting the dual basis of $\left\{\psi_{\lambda}: \lambda \in \nabla\right\}$ for $L^{2}(\mathrm{I})$ as $\left\{\tilde{\psi}_{\lambda}: \lambda \in \nabla\right\}$, furthermore we assume that for some

$$
\mathbb{N} \ni d>m
$$

for all $|\lambda|>0$, for some $\tilde{\omega}_{\lambda} \subseteq \overline{\mathrm{I}}$ with $\operatorname{supp} \tilde{\psi}_{\lambda} \subseteq \tilde{\omega}_{\lambda}$,

(3) $\left|\left\langle\tilde{\psi}_{\lambda}, u\right\rangle_{L^{2}(\mathrm{I})}\right| \lesssim 2^{-|\lambda| d}|u|_{H^{d}\left(\tilde{\omega}_{\lambda}\right)}\left(u \in H^{d}\left(\tilde{\omega}_{\lambda}\right) \cap H_{0, \partial \tilde{\omega}_{\lambda} \cap z}^{m}\left(\tilde{\omega}_{\lambda}\right)\right)$,

(4) $\operatorname{diam} \tilde{\omega}_{\lambda} \approx 2^{-|\lambda|}$,

(5) $\sup _{\ell, j \in \mathbb{N}_{0}} \#\left\{|\lambda|=\ell:\left[j 2^{-\ell},(j+1) 2^{-\ell}\right] \cap \tilde{\omega}_{\lambda} \neq \emptyset\right\}<\infty$.

The fourth and fifth condition mean that the dual wavelets are locally supported and locally finite. From (3), (5) and (1) or (2), one infers that

$$
\begin{aligned}
& \left\|u-\sum_{\{\lambda \in \nabla:|\lambda| \leq \ell\}}\left\langle\tilde{\psi}_{\lambda}, u\right\rangle_{L^{2}(\mathrm{I})} \psi_{\lambda}\right\|_{L^{2}(\mathrm{I})} \lesssim 2^{-d \ell}|u|_{H^{d}(\mathrm{I})} \quad\left(u \in H^{d}(\mathrm{I}) \cap H_{0, z}^{m}(\mathrm{I})\right), \\
& \left\|u-\sum_{\{\lambda \in \nabla:|\lambda| \leq \ell\}}\left\langle\tilde{\psi}_{\lambda}, u\right\rangle_{L^{2}(\mathrm{I})} \psi_{\lambda}\right\|_{H^{m}(\mathrm{I})} \lesssim 2^{-(d-m) \ell}|u|_{H^{d}(\mathrm{I})} \quad\left(u \in H^{d}(\mathrm{I}) \cap H_{0, z}^{m}(\mathrm{I})\right),
\end{aligned}
$$

respectively, meaning that the primal wavelets satisfy a Jackson estimate of order $d$.

For any $m, d \in \mathbb{N}, d>m$, orthogonal or biorthogonal wavelets that satisfy the above conditions have been constructed in many papers. See, e.g., [CDV93] or [DKU99]. 
For $\beta \in[0,1)$ and $\ell \in \mathbb{N}$, following [Nit05], we define

$$
\nabla_{\ell}^{\beta}:=\left\{\lambda \in \nabla:|\lambda| \leq \frac{\ell}{1-\beta}, \tilde{\omega}_{\lambda} \cap\left(0,2^{\frac{\ell-|\lambda|}{\beta}}\right) \neq \emptyset\right\},
$$

see Figure 1. Note that all $\lambda$ 's with levels up to $\ell$ are in this set, and that, for $\beta>0$,

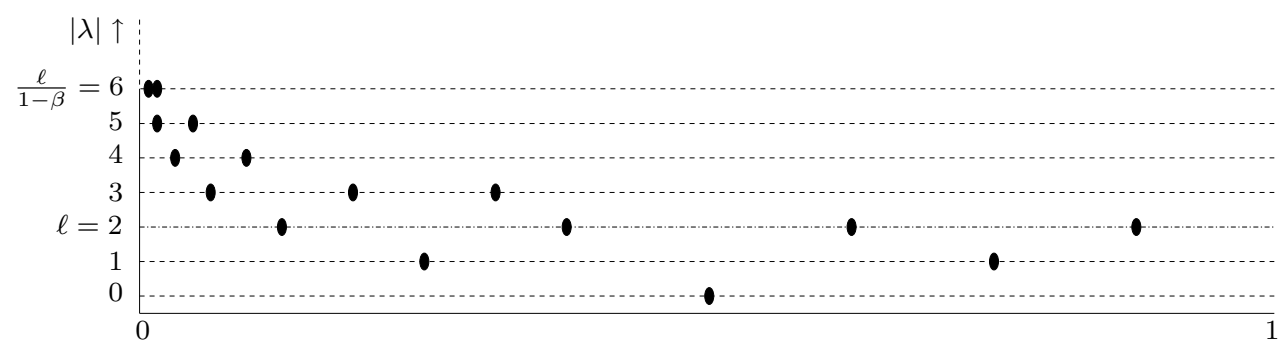

Figure $1 . \nabla_{\ell}^{\beta}$ for $\nabla=\cup_{j \in \mathbb{N}_{0}} 2^{-(j+1)}(2 \mathbb{Z}+1) \cap(0,1)$ where $|\lambda|=j$, $\ell=2, \beta=\frac{2}{3}, \tilde{\omega}_{\lambda}=\left[\lambda-3 \cdot 2^{-|\lambda|}, \lambda+3 \cdot 2^{-|\lambda|}\right] \cap[0,1]$ (corresponds to e.g. biorthogonal spline wavelets of order 2 and with 2 vanishing moments, and $z=\{0,1\})$

it contains also $\lambda$ 's with higher levels, up to $\frac{\ell}{1-\beta}$, when the corresponding $\tilde{\omega}_{\lambda}$ are sufficiently close to zero. For $|\lambda|$ ranging from $\ell$ to $\frac{\ell}{1-\beta}$ (if the latter is an integer), $2^{\frac{\ell-|\lambda|}{\beta}}$ over the 'mesh-size' $2^{-|\lambda|}$ ranges from $2^{\ell}$ to 1 .

On $L^{2}(\mathrm{I})$, we define the projector

$$
P_{\ell}^{\beta}: u \mapsto \sum_{\lambda \in \nabla_{\ell}^{\beta}}\left\langle\tilde{\psi}_{\lambda}, u\right\rangle_{L^{2}(\mathrm{I})} \psi_{\lambda} .
$$

Proposition 2.5. For any $\beta \in[0,1), \# \nabla_{\ell}^{\beta} \approx 2^{\ell}$.

Proof. From the definition of $\nabla_{\ell}^{\beta}$ and (4) and (5), we have

$$
\# \nabla_{\ell}^{\beta} \approx \sum_{j=0}^{\ell} 2^{j}+\sum_{j=\ell+1}^{\left\lfloor\frac{\ell}{1-\beta}\right\rfloor} 2^{\frac{\ell-j}{\beta}} 2^{j} \approx 2^{\ell} .
$$

Estimates for the approximation error for functions from weighted Sobolev spaces are derived in the next theorem and corollary. These estimates were inspired by [Nit05, Lemma 3]. Compared to that result, suboptimal log-factors are removed, and some of the arguments are corrected.

Theorem 2.6. (a). Let $\theta \in[0, d), \beta \in\left(\frac{\theta}{d}, 1\right)$ and $\beta \geq 1-\frac{1}{2 d}$. Then

$$
\left\|u-P_{\ell}^{\beta} u\right\|_{L^{2}(\mathrm{I})} \lesssim 2^{-d \ell}\|u\|_{H_{\theta}^{d}(\mathrm{I})} \quad\left(u \in H_{\theta}^{d}(\mathrm{I}) \cap H_{0, z \cap\{1\}}^{m}(\mathrm{I})\right) .
$$

(b). Let $\theta \in[m, d), \beta \in\left(\frac{\theta-m}{d-m}, 1\right)$ and $\beta \geq 1-\frac{1}{2(d-m)}$. Then

$$
\left\|u-P_{\ell}^{\beta} u\right\|_{H^{m}(\mathrm{I})} \lesssim 2^{-(d-m) \ell}\|u\|_{H_{\theta-m}^{d}(\mathrm{I})} \quad\left(u \in H_{\theta-m}^{d}(\mathrm{I}) \cap H_{0, z}^{m}(\mathrm{I})\right) .
$$


Proof. (a). We write $\nabla \backslash \nabla_{\ell}^{\beta}$ as the disjoint union of sets $\Sigma_{\ell}^{\beta, 1}$ and $\Sigma_{\ell}^{\beta, 2}$, where $\Sigma_{\ell}^{\beta, 1}$ is the set of those $\lambda \in \nabla \backslash \nabla_{\ell}^{\beta}$ with $|\lambda|>\frac{\ell}{1-\beta}$ and $\tilde{\omega}_{\lambda} \cap\left(0,2^{-\frac{\ell}{1-\beta}}\right) \neq \emptyset$. Consider $u \in H_{\theta}^{d}(\mathrm{I}) \cap H_{0, z \cap\{1\}}^{m}(\mathrm{I})$. By $(1)$, we have

$$
\left\|u-P_{\ell}^{\beta} u\right\|_{L^{2}(\mathrm{I})}^{2} \approx \sum_{\lambda \in \Sigma_{\ell}^{\beta, 1}}\left|\left\langle\tilde{\psi}_{\lambda}, u\right\rangle_{L^{2}(\mathrm{I})}\right|^{2}+\sum_{\lambda \in \Sigma_{\ell}^{\beta, 2}}\left|\left\langle\tilde{\psi}_{\lambda}, u\right\rangle_{L^{2}(\mathrm{I})}\right|^{2} .
$$

By (4), the $\left\langle\tilde{\psi}_{\lambda}, u\right\rangle_{L^{2}(\mathrm{I})}$ in the first sum depend only on $u$ restricted to $\left(0, C 2^{-\frac{\ell}{1-\beta}}\right)$, where $C>0$ is some absolute constant. By (1), this sum can therefore be bounded on some absolute multiple of $\|u\|_{L^{2}\left(0, C 2^{-\frac{\ell}{1-\beta}}\right)}^{2}$. Because of the conditions on the parameters $d, \theta$ and $\beta$, there exists a $q \in(2, \infty]$ with $\theta-\left(d-\frac{1}{2}\right)<\frac{1}{q} \leq \frac{1}{2}+d(\beta-1)$. We infer that

$$
\|u\|_{L^{2}\left(0, C 2^{-\frac{\ell}{1-\beta}}\right)}^{2} \leq\left(C 2^{-\frac{\ell}{1-\beta}}\right)^{1-2 / q}\|u\|_{L^{q}(\mathrm{I})}^{2} \lesssim 4^{-\ell d}\|u\|_{L^{q}(\mathrm{I})}^{2} \lesssim 4^{-\ell d}\|u\|_{H_{\theta}^{d}(\mathrm{I})}^{2},
$$

where for the first, second and third inequality, we used $\frac{1}{q} \in\left[0, \frac{1}{2}\right]$ in combination with Hölder's inequality, $\frac{1}{q} \leq \frac{1}{2}+d(\beta-1)$, and $\theta-\left(d-\frac{1}{2}\right)<\frac{1}{q}$, respectively.

By definition of $\Sigma_{\ell}^{\beta, 2}$, and by using (3) and (5), the second sum at the right-hand side of (2.3) can be bounded on some absolute multiple of

$$
\begin{aligned}
& \sum_{j=\ell+1}^{\left\lfloor\frac{\ell}{1-\beta}\right\rfloor} 4^{-j d}|u|_{H^{d}\left(2^{\frac{\ell-j}{\beta}}, 1\right)}^{2}+\sum_{j>\frac{\ell}{1-\beta}} 4^{-j d}|u|_{H^{d}\left(2^{-\frac{\ell}{1-\beta}}, 1\right)}^{2} \\
& \leq \sum_{i=1}^{\infty}\left(\sum_{j=i}^{\infty} 4^{-(\ell+j) d}\right)|u|_{H^{d}\left(2^{-\frac{i}{\beta}}, 2^{-\frac{i-1}{\beta}}\right)}^{2} \\
& \approx 4^{-\ell d} \sum_{i=1}^{\infty} 4^{-i d}|u|_{H^{d}\left(2^{-\frac{i}{\beta}}, 2^{-\frac{i-1}{\beta}}\right)}^{2} \approx 4^{-\ell d}|u|_{H_{\beta d}^{d}(\mathrm{I})}^{2} \lesssim 4^{-\ell d}|u|_{H_{\theta}^{d}(\mathrm{I})}^{2},
\end{aligned}
$$

where we used that $4^{-i d} \approx x^{2 \beta d}$ on $\left(2^{-\frac{i}{\beta}}, 2^{-\frac{i-1}{\beta}}\right)$, and finally that $\theta \leq \beta d$. The estimates for both sums at the right-hand side of (2.3) give the proof of (a).

(b). Consider $u \in H_{\theta-m}^{d}(\mathrm{I}) \cap H_{0, z}^{m}(\mathrm{I})$. Similar to (2.3), by (2) we have

$$
\left\|u-P_{\ell}^{\beta} u\right\|_{H^{m}(\mathrm{I})}^{2} \approx \sum_{\lambda \in \Sigma_{\ell}^{\beta, 1}} 4^{|\lambda| m}\left|\left\langle\tilde{\psi}_{\lambda}, u\right\rangle_{L^{2}(\mathrm{I})}\right|^{2}+\sum_{\lambda \in \Sigma_{\ell}^{\beta, 2}} 4^{|\lambda| m}\left|\left\langle\tilde{\psi}_{\lambda}, u\right\rangle_{L^{2}(\mathrm{I})}\right|^{2} .
$$

Because of (2), the first sum can be bounded on some absolute multiple of $\|u\|_{H^{m}(\mathrm{I})}^{2}$. By (4), we also know that this sum depends only on $u$ restricted to $\left(0, C 2^{-\frac{\ell}{1-\beta}}\right)$ for some absolute constant $C>0$, i.e., that for bounding this sum we may choose $u$ outside this interval at our convenience, as long as it is in $H_{0, z}^{m}(\mathrm{I})$.

There exists a bounded extension operator $E: H^{m}(\mathrm{I}) \rightarrow H_{0,\{2\}}^{m}(0,2)$. By a homogeneity argument, we infer that there exists an extension operator $E_{h}: H^{m}(0, h) \rightarrow$ 
$H_{0,\{2 h\}}^{m}(0,2 h) \subset H_{0,\{1\}}^{m}(\mathrm{I})$ with $\left\|E_{h} v\right\|_{H^{m}(\mathrm{I})}^{2} \lesssim \sum_{k=0}^{m} h^{2(k-m)}|v|_{H^{k}(0, h)}^{2}\left(v \in H^{m}(0, h)\right)$. With $h=C 2^{-\frac{\ell}{1-\beta}}$, we apply this extension operator to $u$.

If $0 \in z$, then $|u|_{H^{k}(0, h)}^{2} \lesssim h^{2(m-k)}|u|_{H^{m}(0, h)}^{2}$, and so for the extended $u$ we find $\|u\|_{H^{m}(\mathrm{I})}^{2} \lesssim|u|_{H^{m}\left(0, C 2^{-\frac{\ell}{1-\beta}}\right)}^{2}$.

If $0 \notin z$, then by $(3)$ the first sum on the right-hand side does not change if we subtract an arbitrary $p \in P_{m-1}$ from $u$. By choosing $p$ suitably, for the resulting $\tilde{u}=u-p$, again we have $|\tilde{u}|_{H^{k}(0, h)}^{2} \lesssim h^{2(m-k)}|\tilde{u}|_{H^{m}(0, h)}^{2}$. We conclude that also in this case the first sum on the right-hand side of (2.4) is bounded by some absolute multiple of $|\tilde{u}|_{H^{m}\left(0, C 2^{-\frac{\ell}{1-\beta}}\right)}^{2}=|u|_{H^{m}\left(0, C 2^{-\frac{\ell}{1-\beta}}\right)}^{2}$.

The remainder of the proof is similar to that of (a). Because of the conditions on the parameters $d, \theta, \beta$ and $m$, there exists a $q \in(2, \infty]$ with $\theta-m-\left(d-m-\frac{1}{2}\right)<$ $\frac{1}{q} \leq \frac{1}{2}+(d-m)(\beta-1)$. By applying Hölder's inequality and Lemma 2.1 we obtain

$$
|u|_{H^{m}\left(0, C 2^{-\frac{\ell}{1-\beta}}\right)}^{2} \leq\left(C 2^{-\frac{\ell}{1-\beta}}\right)^{1-2 / q}\left\|u^{(m)}\right\|_{L^{q}(\mathrm{I})}^{2} \lesssim 4^{-\ell(d-m)}\left\|u^{(m)}\right\|_{H_{\theta-m}^{d-m}(\mathrm{I})}^{2} .
$$

Finally, by (3) and (5), the second sum at the right-hand side of (2.4) can be bounded on some absolute multiple of

$$
\sum_{j=\ell+1}^{\left\lfloor\frac{\ell}{1-\beta}\right\rfloor} 4^{-j(d-m)}|u|_{H^{d}\left(2^{-\frac{\ell-j}{\beta}}, 1\right)}^{2}+\sum_{j>\frac{\ell}{1-\beta}} 4^{-j(d-m)}|u|_{H^{d}\left(2^{\left.-\frac{\ell}{1-\beta}, 1\right)}\right.}^{2} \lesssim 4^{-\ell(d-m)}|u|_{H_{\theta-m}^{d}(\mathrm{I})}^{2}
$$

because of $\theta-m \leq \beta(d-m)$.

Remark 2.7. So far we considered weighted Sobolev spaces with a vanishing weight at $x=0$ appropriate for functions that have a singularity at $x=0$. For functions that may have singularities at both $x=0$ or $x=1$, an suitable adaptation of the $H_{\theta}^{k}(\mathrm{I})$-norm reads as

$$
\|u\|_{H_{\theta}^{k}(\mathrm{I})}:=\left[\sum_{i=0}^{k} \int_{\mathrm{I}}\left|x^{\theta}(1-x)^{\theta} u^{(i)}(x)\right|^{2} d x\right]^{\frac{1}{2}} .
$$

By redefining $\nabla_{\ell}^{\beta}$ as

$$
\nabla_{\ell}^{\beta}:=\left\{\lambda \in \nabla:|\lambda| \leq \frac{\ell}{1-\beta}, \tilde{\omega}_{\lambda} \cap\left(\left(0,2^{\frac{\ell-|\lambda|}{\beta}}\right) \cup\left(1-2^{\frac{\ell-|\lambda|}{\beta}}, 1\right)\right) \neq \emptyset\right\},
$$

it is easily seen that Proposition 2.5 and Theorem 2.6 remain valid, where Theorem 2.6(a) now even reads as

$$
\left\|u-P_{\ell}^{\beta} u\right\|_{L^{2}(\mathrm{I})} \lesssim 2^{-d \ell}\|u\|_{H_{\theta}^{d}(\mathrm{I})} \quad\left(u \in H_{\theta}^{d}(\mathrm{I})\right),
$$

assuming that $\theta \in[0, d), \beta \in\left(\frac{\theta}{d}, 1\right)$ and $\beta \geq 1-\frac{1}{2 d}$.

As follows from (2.1), (2.2) and the uniform $L^{2}(\mathrm{I})$-boundedness of the $P_{\ell}^{\beta}$, if in Theorem 2.6(a) $\theta=0$, or in (b) $\theta=m$, then the corresponding statements are valid for $\beta=0$, i.e., without local refinement, at least when in part (a) additionally 
$u \in H_{0, z}^{m}(\mathrm{I})$ is assumed. In view of this, the additional condition $\beta \geq 1-\frac{1}{2 d}$ or $\beta \geq 1-\frac{1}{2(d-m)}$, besides $\beta>\frac{\theta}{d}$, seems unnatural. Below, for completeness, we will show that indeed they can be omitted, for part (a) at the expense of adding some boundary conditions when $\beta \in\left(\frac{\theta}{d}, 1-\frac{1}{2 d}\right)$. We only consider part (a), since the arguments for part (b) are similar.

Let $\frac{\theta}{d}<1-\frac{1}{2 d}$, i.e., $\theta<d-\frac{1}{2}$, and let $\beta \in\left(\frac{\theta}{d}, 1-\frac{1}{2 d}\right)$. The condition $\beta \geq 1-\frac{1}{2 d}$ was only used for bounding the first sum at the right-hand side of (2.3). For doing so, only the localness of the dual wavelets was used as well as the fact that the primal wavelets form a Riesz basis for $L^{2}(\mathrm{I})$. Alternatively, here we will use the approximation properties of the wavelets given by property (3). In case homogeneous Dirichlet boundary conditions are incorporated in the wavelet construction, it will then be needed that the function $u$ to be approximated also satisfies certain homogeneous Dirichlet boundary conditions.

From (3), (4) and $\left\|\tilde{\psi}_{\lambda}\right\|_{L^{2}(\mathrm{I})} \lesssim 1$, we are going to infer that for $j \in\{1, \ldots, d\}$,

$$
\left|\left\langle\tilde{\psi}_{\lambda}, u\right\rangle_{L^{2}(\mathrm{I})}\right| \lesssim 2^{-|\lambda|\left(j-\frac{1}{2}\right)}|u|_{W^{j, 1}\left(\tilde{\omega}_{\lambda}\right)}
$$

for any sufficiently smooth $u$ that vanishes at order $\min (m, j)$ at $\tilde{\omega}_{\lambda} \cap z$. Indeed, by (4) and a homogeneity argument, it is sufficient to show that $\left|\left\langle\tilde{\psi}_{\lambda}, u\right\rangle_{L^{2}(\mathrm{I})}\right| \lesssim\left\|u^{(j)}\right\|_{L^{1}(\mathrm{I})}$. When $\tilde{\omega}_{\lambda} \cap z=\emptyset$, this follows from $\left\|\tilde{\psi}_{\lambda}\right\|_{L^{2}(\mathrm{I})} \lesssim 1, W^{j, 1} \hookrightarrow L^{2}$, (3), which implies $\tilde{\psi}_{\lambda} \perp_{L^{2}(\mathrm{I})} P_{j-1}$, and the Bramble-Hilbert lemma. When say $\tilde{\omega}_{\lambda} \cap z=\{0\}$, the combination of $\left\|\tilde{\psi}_{\lambda}\right\|_{L^{2}(\mathrm{I})} \lesssim 1$, the embedding $W^{\min (j, m), 1} \hookrightarrow L^{2}$ and the PoincaréFriedrichs' inequality show that $\left|\left\langle\tilde{\psi}_{\lambda}, u\right\rangle_{L^{2}(\mathrm{I})}\right| \lesssim\left\|u^{(\min (j, m))}\right\|_{L^{1}(\mathrm{I})}$. Now for $j>m$, let $p \in P_{j-1}$ be such that $p^{(m)}$ is the Taylor polynomial of $u^{(m)}$ of degree $j-1-m$ at 0 with $p(0)=\cdots=p^{(m-1)}(0)=0$. Then, by $(3),\left|\left\langle\tilde{\psi}_{\lambda}, u\right\rangle_{L^{2}(\mathrm{I})}\right|=\left|\left\langle\tilde{\psi}_{\lambda}, u-p\right\rangle_{L^{2}(\mathrm{I})}\right| \lesssim$ $\left\|u^{(m)}-p^{(m)}\right\|_{L^{1}(\mathrm{I})} \lesssim\left\|u^{(j)}\right\|_{L^{1}(\mathrm{I})}$ as required.

With $\gamma_{v}:=u \mapsto u(v)$ denoting the trace operator, from $W^{1,1}(\mathrm{I}) \hookrightarrow L^{\infty}(\mathrm{I})$ we infer that the closure of the set of smooth functions that vanish at order $\min (m, j)$ at $\tilde{\omega}_{\lambda} \cap z$ in $W^{j, 1}\left(\tilde{\omega}_{\lambda}\right)$ is

$$
\left\{u \in W^{j, 1}\left(\tilde{\omega}_{\lambda}\right): \gamma_{v} D^{r} u=0,0 \leq r \leq \min (m, j)-1, v \in \tilde{\omega}_{\lambda} \cap z\right\},
$$

and thus that (2.5) also holds for all $u$ in this space.

Now we take $j:=\left\lceil d-\theta-\frac{1}{2}\right\rceil \in\{1, \ldots, d\}$. Then, recalling that $\beta \in\left(\frac{\theta}{d}, 1-\frac{1}{2 d}\right)$, there exists a $q \in(1, \infty)$ with $\theta-\left(d-j-\frac{1}{2}\right)<\frac{1}{q} \leq j+\frac{1}{2}-(1-\beta) d$, where the first inequality implies that $H_{\theta}^{d}(\mathrm{I}) \hookrightarrow W^{j, q}(\mathrm{I})$ (see Remark 2.4) $\left(\hookrightarrow W^{j, 1}(\mathrm{I})\right)$, and the second one that $\frac{j+\frac{1}{2}-\frac{1}{q}}{1-\beta} \geq d$. For $u$ in

$$
\tilde{H}_{\theta}^{d, m}(\mathrm{I}):=\left\{u \in H_{\theta}^{d}(\mathrm{I}): \gamma_{v} D^{r} u=0,0 \leq r \leq \min \left(m,\left\lceil d-\theta-\frac{1}{2}\right\rceil\right)-1, v \in z\right\}
$$

from (2.5) and Hölder's inequality we obtain that

$$
\begin{aligned}
\sum_{\lambda \in \Sigma_{\ell}^{\beta, 1}}\left|\left\langle\tilde{\psi}_{\lambda}, u\right\rangle_{L^{2}(\mathrm{I})}\right|^{2} & \lesssim 4^{-\frac{\ell}{1-\beta}\left(j-\frac{1}{2}\right)}|u|_{W^{j, 1}\left(0, C 2^{-\frac{\ell}{1-\beta}}\right)}^{2} \\
& \leq 4^{-\frac{\ell}{1-\beta}\left(j+\frac{1}{2}-\frac{1}{q}\right)}|u|_{W^{j, q}(\mathrm{I})}^{2} \lesssim 4^{-\ell d}\|u\|_{H_{\theta}^{d}(\mathrm{I})}^{2}
\end{aligned}
$$


being the upper bound that was required.

Noting that for $\theta \geq d-\frac{1}{2}$, the set of additional conditions incorporated in the definition of $\tilde{H}_{\theta}^{d, m}(\mathrm{I})$ is empty, and thus that $\tilde{H}_{\theta}^{d, m}(\mathrm{I})=H_{\theta}^{d}(\mathrm{I})$, we have shown the first statement of the following Corollary. As noted before, the proof of the second part is similar.

Corollary 2.8. (a). Let $\theta \in[0, d)$ and $\beta \in\left(\frac{\theta}{d}, 1\right)$. Then

$$
\left\|u-P_{\ell}^{\beta} u\right\|_{L^{2}(\mathrm{I})} \lesssim 2^{-d \ell}\|u\|_{H_{\theta}^{d}(\mathrm{I})} \quad\left(u \in \tilde{H}_{\theta}^{d, m}(\mathrm{I})\right) .
$$

(b). Let $\theta \in[m, d)$ and $\beta \in\left(\frac{\theta-m}{d-m}, 1\right)$. Then

$$
\left\|u-P_{\ell}^{\beta} u\right\|_{H^{m}(\mathrm{I})} \lesssim 2^{-(d-m) \ell}\|u\|_{H_{\theta-m}^{d}(\mathrm{I})} \quad\left(u \in H_{\theta-m}^{d}(\mathrm{I}) \cap H_{0, z}^{m}(\mathrm{I})\right) .
$$

To indicate the dependence on the prescribed Dirichlet boundary $z \subseteq \partial \mathrm{I}$ of the wavelets $\psi_{\lambda}$, the dual wavelets $\tilde{\psi}_{\lambda}$, the sets $\tilde{\omega}_{\lambda} \supseteq \operatorname{supp} \tilde{\psi}_{\lambda}$, the index sets $\nabla$ and $\nabla_{\ell}^{\beta}$, the projector $P_{\ell}^{\beta}$, and the space $\tilde{H}_{\theta}^{d, m}(\mathrm{I})$, from now on we will denote them as

$$
\psi_{\lambda}^{(z)}, \tilde{\psi}_{\lambda}^{(z)}, \tilde{\omega}_{\lambda}^{(z)}, \nabla^{(z)}, \nabla_{\ell}^{\beta, z}, P_{\ell}^{\beta, z}, \tilde{H}_{\theta, z}^{d, m}(\mathrm{I})
$$

respectively.

\section{Tensor products of Hilbert spaces}

We recall some facts and give some new results about tensor products of Hilbert spaces, in particular (weighted) Sobolev spaces. Although for notational convenience, we start with formulating some results for tensor products of two spaces, these results obviously generalize to multiple products.

Proposition 3.1. For $i \in\{1,2\}$, let $\Omega_{i}$ be domains in $\mathbb{R}^{n_{i}}$ that satisfy the uniform cone property (cf. [Ada75, Th. 4.32]). Then for $k \in \mathbb{N}_{0}$,

$$
H^{k}\left(\Omega_{1} \times \Omega_{2}\right)=H^{k}\left(\Omega_{1}\right) \otimes L^{2}\left(\Omega_{2}\right) \cap L^{2}\left(\Omega_{1}\right) \otimes H^{k}\left(\Omega_{2}\right) .
$$

Proof. For $i \in\{1,2\}$, let $\Sigma_{i}$ be a Riesz basis for $L^{2}\left(\Omega_{i}\right)$, formally viewed as a column vector, such that for some invertible diagonal matrix $\mathbf{D}_{i}$ and $0 \leq j \leq k, \mathbf{D}_{i}^{-j / k} \Sigma_{i}$ is a Riesz basis for $H^{j}\left(\Omega_{i}\right)$, meaning that $\sum_{|\alpha| \leq j}\left\langle\partial^{\alpha} \Sigma_{i}, \partial^{\alpha} \Sigma_{i}\right\rangle_{L^{2}\left(\Omega_{i}\right)} \approx \mathbf{D}_{i}^{2 j / k}$. Later we will show that such bases exist.

Using that $L^{2}\left(\Omega_{1} \times \Omega_{2}\right)=L^{2}\left(\Omega_{1}\right) \otimes L^{2}\left(\Omega_{2}\right)$, and that the tensor product of Riesz bases is a Riesz basis for the tensor product, each $u \in L^{2}\left(\Omega_{1} \times \Omega_{2}\right)$ has a unique expansion $u=\mathbf{c}^{\top} \Sigma_{1} \otimes \Sigma_{2}$ where $\mathbf{c}=\left(c_{\sigma_{1} \sigma_{2}}\right)_{\sigma_{1} \in \Sigma_{1}, \sigma_{2} \in \Sigma_{2}} \in \ell^{2}\left(\Sigma_{1} \times \Sigma_{2}\right)$. We have that

$$
\begin{aligned}
& \sum_{\left|\alpha_{1}\right|+\left|\alpha_{2}\right| \leq k}\left\|\partial_{1}^{\alpha_{1}} \partial_{2}^{\alpha_{2}} u\right\|_{L^{2}\left(\Omega_{1} \times \Omega_{2}\right)}^{2} \\
& =\sum_{\left|\alpha_{1}\right|+\left|\alpha_{2}\right| \leq k} \mathbf{c}^{\top}\left\langle\partial^{\alpha_{1}} \Sigma_{1}, \partial^{\alpha_{1}} \Sigma_{1}\right\rangle_{L^{2}\left(\Omega_{1}\right)} \otimes\left\langle\partial^{\alpha_{2}} \Sigma_{2}, \partial^{\alpha_{2}} \Sigma_{2}\right\rangle_{L^{2}\left(\Omega_{2}\right)} \mathbf{c} \\
& \bar{\sim} \mathbf{c}^{\top}\left(\mathbf{D}_{1}^{2} \otimes \mathrm{Id}+\mathrm{Id} \otimes \mathbf{D}_{2}^{2}\right) \mathbf{c} \approx\|u\|_{H^{k}\left(\Omega_{1}\right) \otimes L^{2}\left(\Omega_{2}\right)}^{2}+\|u\|_{L^{2}\left(\Omega_{1}\right) \otimes H^{k}\left(\Omega_{2}\right)}^{2},
\end{aligned}
$$


where for the one but last " $\sim$ " we used that for $0<j<k, \mathbf{D}_{1}^{2 j / k} \otimes \mathbf{D}_{2}^{2(k-j) / k} \leq$ $\mathbf{D}_{1}^{2} \otimes \mathrm{Id}+\mathrm{Id} \otimes \mathbf{D}_{2}^{2}$.

The existence of the aforementioned bases $\Sigma_{i}$ has still to be shown. Let $V_{0} \subset V_{1} \subset$ $\cdots \subset L_{2}\left(\Omega_{i}\right)$ be such that for some $d, \gamma>0, \inf _{v_{\ell} \in V_{\ell}}\left\|u-v_{\ell}\right\|_{L^{2}\left(\Omega_{i}\right)} \lesssim 2^{-\ell d}\|u\|_{H^{d}\left(\Omega_{i}\right)}$ $\left(u \in H^{d}\left(\Omega_{i}\right)\right.$ ) (Jackson estimate) and for all $s<\gamma,\|\cdot\|_{H^{s}\left(\Omega_{i}\right)} \lesssim 2^{\ell s}\|\cdot\|_{L^{2}\left(\Omega_{i}\right)}$ on $V_{\ell}$ (Bernstein estimate). With $V_{-1}:=\{0\}$, let $\Psi_{\ell}$ be an $L_{2}\left(\Omega_{i}\right)$-orthonormal basis for $V_{\ell} \cap V_{\ell-1}^{\perp_{L^{2}\left(\Omega_{i}\right)}}$. Then for $|s|<\min (\gamma, d), \cup_{\ell \in \mathbb{N}_{0}} 2^{-s \ell} \Psi_{\ell}$ is a Riesz basis for $H^{s}\left(\Omega_{i}\right)$ (cf. e.g. [Dah96]), and so when $\min (\gamma, d)>k, \Sigma_{i}:=\cup_{\ell \in \mathbb{N}_{0}} \Psi_{\ell}$ satisfies the assumptions.

When $\Omega_{i}=\mathbb{R}^{n_{i}}$, for any $d \in \mathbb{N}$ and with $\gamma:=d-\frac{1}{2}$ such a ladder of spaces, denoted as $\left(V_{\ell}^{\mathbb{R}^{n_{i}}}\right)_{\ell}$, can be constructed as a sequence of spline spaces with respect to nested dyadic partitions. Then, since the restriction of a function on $\mathbb{R}^{n_{i}}$ to $\Omega_{i}$ is a bounded operator from $H^{s}\left(\mathbb{R}^{n_{i}}\right) \rightarrow H^{s}\left(\Omega_{i}\right)$, the sequence $\left(V_{\ell}\right)_{\ell}$ defined by $V_{\ell}:=\left.V_{\ell}^{\mathbb{R}^{n_{i}}}\right|_{\Omega_{i}}$ satisfies the corresponding Bernstein inequality on $\Omega_{i}$. Thanks to the uniform cone condition, there exists a bounded extension operator $E: H^{d}\left(\Omega_{i}\right) \rightarrow H^{d}\left(\mathbb{R}^{n_{i}}\right)$ ([CZ52]). For $u \in H^{d}\left(\Omega_{i}\right)$, we have

$$
\inf _{v_{j} \in V_{\ell}^{\mathbb{R}^{n_{i}}}}\left\|u-\left.v_{j}\right|_{\Omega_{i}}\right\|_{L^{2}\left(\Omega_{i}\right)} \leq \inf _{v_{j} \in V_{\ell}^{\mathbb{R}_{i}}}\left\|E u-v_{j}\right\|_{L^{2}\left(\mathbb{R}^{\left.n_{i}\right)}\right.} \lesssim 2^{-\ell d}\|E u\|_{H^{d}\left(\mathbb{R}^{\left.n_{i}\right)}\right.} \lesssim\|u\|_{H^{d}\left(\Omega_{i}\right)},
$$

i.e., the corresponding Jackson estimate is valid on $\Omega_{i}$, with which the existence of $\Sigma_{i}$ is demonstrated.

Theorem 3.2. Let $H, K, Z$ be separable Hilbert spaces, and let $G: H \rightarrow Z$ be a linear map that is bounded and onto. Then

$$
\{u \in H \otimes K:(G \otimes \mathrm{Id}) u=0\}=\{v \in H: G v=0\} \otimes K .
$$

Proof. Since $G$ is bounded, $\tilde{H}:=\{v \in H: G v=0\}$ is closed, and so $H$ is the direct sum of the Hilbert spaces $\tilde{H}$ and $\tilde{H}^{\perp}$.

Let $\Xi, \Sigma$ and $\Upsilon$ be orthonormal bases for $\tilde{H}, \tilde{H}^{\perp}$ and $K$, respectively. Then $\Xi \otimes \Upsilon$, $\Sigma \otimes \Upsilon$ and $(\Xi \cup \Sigma) \otimes \Upsilon$ are orthonormal bases for $\tilde{H} \otimes K, \tilde{H}^{\perp} \otimes K$ and $H \otimes K$, respectively. Any $u \in H \otimes K$ has a unique expansion $u=\mathbf{c}^{\top} \Xi \otimes \Upsilon+\mathbf{d}^{\top} \Sigma \otimes \Upsilon$, where $\mathbf{c} \in \ell^{2}(\Xi \times \Upsilon), \mathbf{d} \in \ell^{2}(\Sigma \times \Upsilon)$. The condition $(G \otimes \mathrm{Id}) u=0$ means that $\mathbf{d}^{\top} G(\Sigma) \otimes \Upsilon=0$.

The linear mapping $\left.G\right|_{\tilde{H}^{\perp}}: \tilde{H}^{\perp} \rightarrow Z$ is onto, bounded and injective. Since $\tilde{H}^{\perp}$ and $Z$ are Hilbert spaces, the open mapping theorem shows that $\left.G\right|_{\tilde{H}^{\perp}}$ is boundedly invertible. We conclude that $G(\Sigma)$ is a Riesz basis for $Z$ and thus that $G(\Sigma) \otimes \Upsilon$ is a Riesz basis for $Z \otimes K$. So $\mathbf{d}^{\top} G(\Sigma) \otimes \Upsilon=0$ implies $\mathbf{d}=0$, which shows that $\Xi \otimes \Upsilon$ is an (orthonormal) basis for $\{u \in H \otimes K:(G \otimes \mathrm{Id}) u=0\}$, and thus completes the proof.

Corollary 3.3. Let $k \in \mathbb{N}$, and for $i \in\{1,2\}$, let $\Omega_{i}$ be a domain in $\mathbb{R}^{n_{i}}$ with a $C^{k-1,1}$ boundary. Then

$$
H_{0}^{k}\left(\Omega_{1} \times \Omega_{2}\right)=H_{0}^{k}\left(\Omega_{1}\right) \otimes L^{2}\left(\Omega_{2}\right) \cap L^{2}\left(\Omega_{1}\right) \otimes H_{0}^{k}\left(\Omega_{2}\right) .
$$

Proof. With $\gamma_{i}$ denoting the trace operator on $\partial \Omega_{i}$, the mapping $G_{i}: H^{k}\left(\Omega_{i}\right) \rightarrow$ $\prod_{j=0}^{k-1} H^{k-j-\frac{1}{2}}\left(\partial \Omega_{i}\right): u \mapsto\left(\gamma_{i} u, \gamma_{i} \frac{\partial u}{\partial n}, \ldots, \gamma_{i} \frac{\partial^{k-1} u}{\partial n^{k-1}}\right)$ is bounded and onto, the latter 
because of $\partial \Omega_{i} \in C^{k-1,1}$ (see, e.g., [Gri85, Th. 1.5.1.1]). Using

$$
\begin{aligned}
& H_{0}^{k}\left(\Omega_{1} \times \Omega_{2}\right)=\left\{u \in H^{k}\left(\Omega_{1} \times \Omega_{2}\right):\left(G_{1} \otimes \mathrm{Id}\right) u=0,\left(\operatorname{Id} \otimes G_{2}\right) u=0\right\}= \\
& \left\{u \in H^{k}\left(\Omega_{1}\right) \otimes L^{2}\left(\Omega_{2}\right):\left(G_{1} \otimes \mathrm{Id}\right) u=0\right\} \cap\left\{u \in L^{2}\left(\Omega_{1}\right) \otimes H^{k}\left(\Omega_{2}\right):\left(\operatorname{Id} \otimes G_{2}\right) u=0\right\}
\end{aligned}
$$

by Proposition 3.1, and $H_{0}^{k}\left(\Omega_{i}\right)=\left\{u \in H^{k}\left(\Omega_{i}\right): G_{i} u=0\right\}$, an application of Theorem 3.2 completes the proof.

Results related to Corollary 3.3 can be found in [Dau88, (AC.7)], [GO95], [Hoc01]. The technique employed here allows us also to deal with weighted Sobolev spaces that incorporate boundary conditions.

Applications of Theorem 3.2 specific for this work involve multiple tensor products of (weighted) Sobolev spaces on I. With, for $n \in \mathbb{N}$,

$$
\square:=(0,1)^{n} \text {, }
$$

let $\Gamma$ be a union of $(n-1)$-dimensional faces of $\bar{\square}$, i.e., $\Gamma=\cup_{i=1}^{n} \overline{\mathrm{I}}^{i-1} \times z_{i} \times \overline{\mathrm{I}}^{n-i}$ for some $z_{i} \subseteq \partial \mathrm{I}$, see Figure 2 for an illustration.

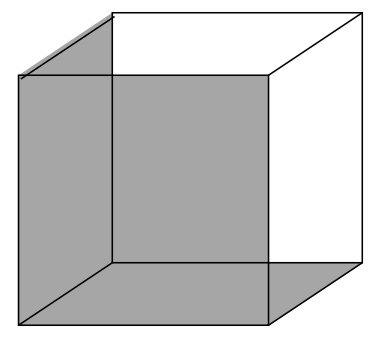

FiguRE 2. $\Gamma$ for $n=3$ and $z_{i}=\{0\}$ for $1 \leq i \leq n$.

As a special case of Proposition 3.1 (for multiple tensor products), we have

$$
H^{m}(\square)=H^{m}(\mathrm{I}) \otimes L^{2}(\mathrm{I}) \otimes \cdots \otimes L^{2}(\mathrm{I}) \cap \cdots \cap L^{2}(\mathrm{I}) \otimes \cdots \otimes L^{2}(\mathrm{I}) \otimes H^{m}(\mathrm{I}),
$$

Furthermore, we have

$$
H_{0, \Gamma}^{m}(\square)=H_{0, z_{1}}^{m}(\mathrm{I}) \otimes L^{2}(\mathrm{I}) \otimes \cdots \otimes L^{2}(\mathrm{I}) \cap \cdots \cap L^{2}(\mathrm{I}) \otimes \cdots \otimes L^{2}(\mathrm{I}) \otimes H_{0, z_{n}}^{m}(\mathrm{I}) .
$$

To see the latter, for $k \in \mathbb{N}$, and $1 \leq i \leq n$, let us put

$$
G_{i, k}:=u \mapsto \prod_{v \in z_{i}} \prod_{r=0}^{k-1} \gamma_{v} D^{r} u
$$

Then, since $G_{i, m}: H^{m}(\mathrm{I}) \rightarrow \mathbb{R}^{m \cdot \# z_{i}}$ is bounded and onto, and

$$
\begin{aligned}
& H_{0, \Gamma}^{m}(\square)=\left\{u \in H^{m}(\square):\left(\operatorname{Id} \otimes \cdots \otimes G_{i, m} \otimes \cdots \otimes \mathrm{Id}\right) u=0,1 \leq i \leq n\right\} \\
& =\cap_{i=1}^{n}\{u \in L^{2}(\mathrm{I}) \otimes \cdots \otimes \underbrace{H^{m}(\mathrm{I})}_{\text {ith position }} \otimes \cdots \otimes L^{2}(\mathrm{I}):\left(\mathrm{Id} \otimes \cdots \otimes G_{i, m} \otimes \cdots \otimes \mathrm{Id}\right) u=0\},
\end{aligned}
$$

the result follows from an application of Theorem 3.2. 
Similarly, we have that

$$
\begin{aligned}
& \otimes_{i=1}^{n} \tilde{H}_{\theta, z_{i}}^{d, m}(\mathrm{I})= \\
& \quad\left\{u \in \otimes_{i=1}^{n} H_{\theta}^{d}(\mathrm{I}): \partial_{n}^{r} u=0 \text { on } \Gamma, 0 \leq r \leq \min \left(m,\left\lceil d-\theta-\frac{1}{2}\right\rceil\right)-1\right\} .
\end{aligned}
$$

Indeed, for $\theta<d-\frac{1}{2}$ putting $k:=\min \left(m,\left\lceil d-\theta-\frac{1}{2}\right\rceil\right), G_{i, k}: H_{\theta}^{d}(\mathrm{I}) \rightarrow \mathbb{R}^{k \cdot \# z_{i}}$ is bounded (cf. arguments that led to Corollary 2.8) and onto, and the result follows from Theorem 3.2 using the space at the right hand side of (3.3) being equal to $\left\{u \in \otimes_{i=1}^{n} H_{\theta}^{d}(\mathrm{I}):\left(\operatorname{Id} \otimes \cdots \otimes G_{i, k} \otimes \cdots \mathrm{Id}\right) u=0,1 \leq i \leq n\right\}$ and the definition of $\tilde{H}_{\theta, z_{i}}^{d, m}(\mathrm{I})$ in $(2.6)$.

Finally, we note that for $\theta \in[0, d)$,

$$
\cap_{p=1}^{n} \otimes_{i=1}^{n} H_{\theta-\delta_{i p} \min (m, \theta)}^{d}(\mathrm{I}) \cap H_{0, \Gamma}^{m}(\square)=\cap_{p=1}^{n} \otimes_{i=1}^{n} \mathcal{W}_{i p}(\mathrm{I}),
$$

where

$$
\mathcal{W}_{p p}=H_{\max (0, \theta-m)}^{d}(\mathrm{I}) \cap H_{0, z_{p}}^{m}(\mathrm{I})
$$

and for $i \neq p$,

$$
\mathcal{W}_{i p}=H_{\theta}^{d}(\mathrm{I}) \quad \text { or } \quad \mathcal{W}_{i p}=\tilde{H}_{\theta, z_{i}}^{d, m}(\mathrm{I})
$$

Indeed, with the first option $\mathcal{W}_{i p}=H_{\theta}^{d}(\mathrm{I})$ for $i \neq p$, the result follows from Theorem 3.2 by writing the space at the left hand side of $(3.4 \mathrm{a})$ as

$$
\cap_{p=1}^{n}\left\{u \in \otimes_{i=1}^{n} H_{\theta-\delta_{i p} \min (m, \theta)}^{d}(\mathrm{I}):\left(\mathrm{Id} \otimes \cdots \otimes G_{p, m} \otimes \cdots \otimes \mathrm{Id}\right) u=0\right\} .
$$

Here we used that $\cap_{p=1}^{n} \otimes_{i=1}^{n} H_{\theta-\delta_{i p} \min (m, \theta)}^{d}(\mathrm{I}) \hookrightarrow H^{m}(\square)$, which follows from (3.1) and $H_{\theta}^{d}(\mathrm{I}) \hookrightarrow L^{2}(\mathrm{I})$ and $H_{\max (0, \theta-m)}^{d}(\mathrm{I}) \hookrightarrow H^{m}(\mathrm{I})$ by $\theta<d$ (cf. Lemma 2.1 and Remark 2.4).

With the second option $\mathcal{W}_{i p}=\tilde{H}_{\theta, z_{i}}^{d, m}(\mathrm{I})$ for $i \neq p$, the result follows by "copying" certain boundary conditions, i.e., with $k:=\min \left(m,\left\lceil d-\theta-\frac{1}{2}\right\rceil\right)$, by writing the space at the left hand side of $(3.4 \mathrm{a})$ as

$$
\begin{array}{r}
\cap_{p=1}^{n}\left\{u \in \otimes_{i=1}^{n} H_{\theta-\delta_{i p} \min (m, \theta)}^{d}(\mathrm{I}):\left(\mathrm{Id} \otimes \cdots \otimes G_{p, m} \otimes \cdots \otimes \mathrm{Id}\right) u=0\right. \\
\left.\left(\mathrm{Id} \otimes \cdots \otimes G_{i, k} \otimes \cdots \otimes \mathrm{Id}\right) u=0(1 \leq i \leq n, i \neq p)\right\} .
\end{array}
$$

\section{Optimal WaVElet approximation of Singular FUnCtions Using SPARSE PRODUCTS OF LOCALLY REFINED INDEX SETS}

As shown in [GO95], from $L^{2}(\square)=\otimes_{i=1}^{n} L^{2}(\mathrm{I}),(3.2)$ and (1), (2), we have that

$$
\left\{\boldsymbol{\psi}_{\boldsymbol{\lambda}}:=\psi_{\lambda_{1}}^{\left(z_{1}\right)} \otimes \cdots \otimes \psi_{\lambda_{1}}^{\left(z_{n}\right)}: \boldsymbol{\lambda} \in \boldsymbol{\nabla}:=\prod_{i=1}^{n} \nabla^{\left(z_{i}\right)}\right\}, \quad\left\{\left(\sum_{i=1}^{n} 4^{\left|\lambda_{i}\right|}\right)^{-m / 2} \boldsymbol{\psi}_{\boldsymbol{\lambda}}: \boldsymbol{\lambda} \in \boldsymbol{\nabla}\right\}
$$

are Riesz bases for $L^{2}(\square)$ and $H_{0, \Gamma}^{m}(\square)$, respectively. 
For $\beta \in[0,1), \nu \geq 1$ and $L \in \mathbb{N}_{0}$, and with $\nabla_{-1}^{\beta, z_{i}}:=\emptyset$, we define

$$
\begin{aligned}
\nabla_{L, \nu}^{\beta} & :=\left\{\prod_{i=1}^{n} \nabla_{\ell_{i}}^{\beta, z_{i}} \backslash \nabla_{\ell_{i}-1}^{\beta, z_{i}}: \ell \in \mathbb{N}_{0}^{n}, \nu\|\ell\|_{1}+(1-\nu)\|\ell\|_{\infty} \leq L\right\} \\
& =\bigcup_{p=1}^{n}\left\{\prod_{i=1}^{n} \nabla_{\ell_{i}}^{\beta, z_{i}} \backslash \nabla_{\ell_{i}-1}^{\beta, z_{i}}: \ell \in \mathbb{N}_{0}^{n}, \ell_{p}+\nu\left(\|\ell\|_{1}-\ell_{p}\right) \leq L\right\},
\end{aligned}
$$

see Figure 3. The index set $\nabla_{L, 1}^{0}$ underlies the well-known sparse grid, also called

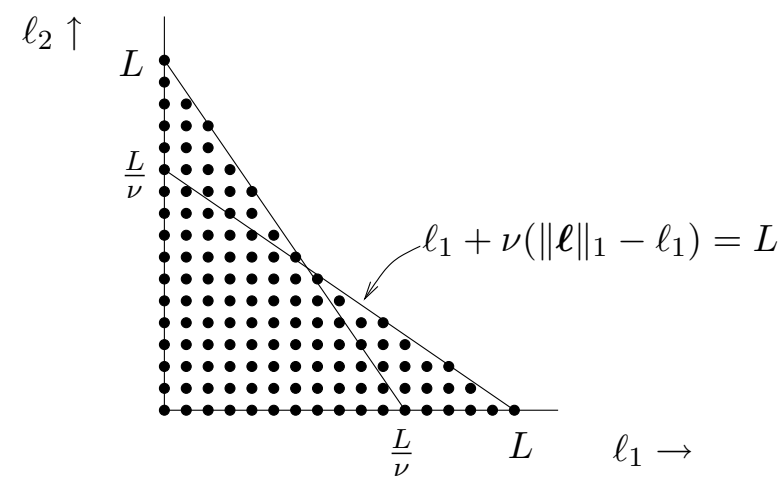

Figure 3. $\left\{\boldsymbol{\ell} \in \mathbb{N}_{0}^{n}: \nu\|\ell\|_{1}+(1-\nu)\|\ell\|_{\infty} \leq L\right\}$ for $n=2$ and $\nu=\frac{16}{11}$.

hyperbolic wavelet approximation, see, e.g. [Zen91, BG04, DKT98]. By considering $\boldsymbol{\nabla}_{L, 1}^{\beta}$ for $\beta>0$, in [Nit05] this type of approximation was combined with (product type) local refinements towards $\partial \square$.

As shown in [GK00], when measuring approximation errors in Sobolev spaces with positive smoothness indices, sparse-grid approximation rates can be realized with even smaller spaces ("optimized" sparse grids), with which truly optimal convergence rates are realized. With the introduction of the index sets $\nabla_{L, \nu}^{\beta}$ for $\nu>1$, we combine this idea with that of local refinement. With that, we will be be able to remove suboptimal log-factors from the corresponding estimates from [Nit05].

The following elementary lemma will be used a couple of times.

Lemma 4.1. For any $\nu>0, \alpha \geq 0$,

$$
\sum_{\left\{\boldsymbol{\ell} \in \mathbb{N}_{0}^{n}: \ell_{p}+\nu\left(\|\boldsymbol{\ell}\|_{1}-\ell_{p}\right) \in[j, j+1]\right\}} 2^{-\alpha\left(\|\boldsymbol{\ell}\|_{1}-\ell_{p}\right)} \approx\left\{\begin{array}{cl}
j^{n-1} & \text { if } \alpha=0, \\
1 & \text { if } \alpha>0,
\end{array}\right.
$$

where the constant factors absorbed by the " $\sim$ " symbol may depend on $\nu, \alpha$ and $n$ (but not on $j$ ).

On $L^{2}(\square)$, we define the projector

$$
\mathbf{P}_{L, \nu}^{\beta}: u=\sum_{\boldsymbol{\lambda} \in \boldsymbol{\nabla}} u_{\boldsymbol{\lambda}} \psi_{\boldsymbol{\lambda}} \mapsto \sum_{\boldsymbol{\lambda} \in \nabla_{L, \nu}^{\beta}} u_{\boldsymbol{\lambda}} \psi_{\boldsymbol{\lambda}}
$$


Proposition 4.2. Let $\beta \in[0,1)$. Then for any $\nu \geq 1, \# \nabla_{L, \nu}^{\beta} \approx\left\{\begin{array}{cl}L^{n-1} 2^{L} & \text { if } \nu=1, \\ 2^{L} & \text { if } \nu>1 .\end{array}\right.$ Proof. Since by Proposition 2.5, \# $\left(\nabla_{\ell_{i}}^{\beta, z_{i}} \backslash \nabla_{\ell_{i}-1}^{\beta, z_{i}}\right) \approx 2^{\ell_{i}}$, the proof follows from

$$
\begin{aligned}
& \sum_{j=0}^{L} \sum_{\left\{\ell: \ell_{p}+\nu\left(\|\ell\|_{1}-\ell_{p}\right) \in[j, j+1]\right\}} 2^{\|\ell\|_{1}}=\sum_{j=0}^{L} \sum_{\left\{\ell: \ell_{p}+\nu\left(\|\ell\|_{1}-\ell_{p}\right) \in[j, j+1]\right\}} 2^{\ell_{p}+\nu\left(\|\ell\|_{1}-\ell_{p}\right)} 2^{(1-\nu)\left(\|\ell\|_{1}-\ell_{p}\right)} \\
& \approx \sum_{j=0}^{L} 2^{j} \times\left\{\begin{array}{cl}
j^{n-1} & \text { if } \nu=1 \\
1 & \text { if } \nu>1
\end{array}\right\}=\left\{\begin{array}{cl}
L^{n-1} 2^{L} & \text { if } \nu=1, \\
2^{L} & \text { if } \nu>1,
\end{array}\right.
\end{aligned}
$$

by an application of Lemma 4.1.

We have the following estimates for the approximation error:

Theorem 4.3. Let $\theta \in[0, d)$ and $\beta \in\left(\frac{\theta}{d}, 1\right)$. Then

$$
\left\|u-\mathbf{P}_{L, 1}^{\beta} u\right\|_{L^{2}(\square)} \lesssim L^{\frac{n-1}{2}} 2^{-d L}\|u\|_{\otimes_{i=1}^{n} H_{\theta}^{d}(\mathrm{I})}
$$

for all $u$ in

$$
\left\{u \in \otimes_{i=1}^{n} H_{\theta}^{d}(\mathrm{I}): \partial_{n}^{r} u=0 \text { on } \Gamma, 0 \leq r \leq \min \left(m,\left\lceil d-\theta-\frac{1}{2}\right\rceil\right)-1\right\} ;
$$

and for $\nu<\frac{d}{d-m}$,

$$
\left\|u-\mathbf{P}_{L, \nu}^{\beta} u\right\|_{H^{m}(\square)} \lesssim 2^{-(d-m) L} \sqrt{\sum_{p=1}^{n}\|u\|_{\otimes_{i=1}^{n} H_{\theta-\delta_{p i} \min (m, \theta)}^{2}(\mathrm{I})}^{2}}
$$

for all $u \in \cap_{p=1}^{n} \otimes_{i=1}^{n} H_{\theta-\delta_{p i} \min (m, \theta)}^{d}(\mathrm{I}) \cap H_{0, \Gamma}^{m}(\square)$.

Proof. (a). Setting $P_{-1}^{\beta, z_{i}}:=0$, the mapping $u \mapsto \sum_{\boldsymbol{\lambda} \in \prod_{i=1}^{n} \nabla_{\ell_{i}}^{\beta, z_{i}} \backslash \nabla_{\ell_{i}-1}^{\beta, z_{i}}} u_{\boldsymbol{\lambda}} \boldsymbol{\psi}_{\boldsymbol{\lambda}}$ is equal to $\otimes_{i=1}^{n}\left(P_{\ell_{i}}^{\beta, z_{i}}-P_{\ell_{i}-1}^{\beta, z_{i}}\right)$, so that

$$
\mathrm{I}-\mathbf{P}_{L, \nu}^{\beta}=\sum_{\left\{\ell: \nu\|\ell\|_{1}+(1-\nu)\|\ell\|_{\infty}>L\right\}} \otimes_{i=1}^{n}\left(P_{\ell_{i}}^{\beta, z_{i}}-P_{\ell_{i}-1}^{\beta, z_{i}}\right) .
$$

From $\left\{\boldsymbol{\psi}_{\boldsymbol{\lambda}}: \boldsymbol{\lambda} \in \boldsymbol{\nabla}\right\}$ being a Riesz basis for $L^{2}(\square)$, we even have $\left\|u-\mathbf{P}_{L, 1}^{\beta} u\right\|_{L^{2}(\square)}^{2} \bar{\sim}$ $\sum_{\left\{\ell:\|\ell\|_{1}>L\right\}}\left\|\otimes_{i=1}^{n}\left(P_{\ell_{i}}^{\beta, z_{i}}-P_{\ell_{i}-1}^{\beta, z_{i}}\right) u\right\|_{L^{2}(\square)}^{2}$. The combination of Corollary 2.8(a) and a tensor product argument show that

$$
\left\|\otimes_{i=1}^{n}\left(P_{\ell_{i}}^{\beta, z_{i}}-P_{\ell_{i}-1}^{\beta, z_{i}}\right) u\right\|_{L^{2}(\square)} \lesssim 2^{-d\|\ell\|_{1}}\|u\|_{\otimes_{i=1}^{n} H_{\theta}^{d}(\mathrm{I})} \quad\left(u \in \otimes_{i=1}^{n} \tilde{H}_{\theta, z_{i}}^{d, m}(\mathrm{I})\right) .
$$

From (3.3) we know that $\otimes_{i=1}^{n} \tilde{H}_{\theta, z_{i}}^{d, m}(\mathrm{I})$ is equal to the space in (4.1). An application of Lemma 4.1 together with $\sum_{j=L+1}^{\infty} 4^{-d j} j^{n-1} \approx 4^{-d L} L^{n-1}$ completes the proof of part (a).

(b). Using $\theta<d$, in view of (3.4a), (3.2), (4.2), and of the inclusions

$$
\left\{\boldsymbol{\ell}: \nu\|\boldsymbol{\ell}\|_{1}+(1-\nu)\|\boldsymbol{\ell}\|_{\infty}>L\right\} \subset\left\{\boldsymbol{\ell}: \ell_{p}+\nu\left(\|\boldsymbol{\ell}\|_{1}-\ell_{p}\right)>L\right\} \quad(p=1, \ldots, n),
$$


w.l.o.g. it suffices to show that

$$
\begin{aligned}
& \sum_{\left\{\ell: \ell_{1}+\nu\left(\|\ell\|_{1}-\ell_{1}\right)>L\right\}}\left\|\otimes_{i=1}^{n}\left(P_{\ell_{i}}^{\beta, z_{i}}-P_{\ell_{i}-1}^{\beta, z_{i}}\right) u\right\|_{H^{m}(\mathrm{I}) \otimes L^{2}(\mathrm{I}) \otimes \cdots \otimes L^{2}(\mathrm{I})} \\
& \lesssim 2^{-(d-m) L}\|u\|_{H_{\max (0, \theta-m)}^{d}(\mathrm{I}) \otimes H_{\theta}^{d}(\mathrm{I}) \otimes \cdots \otimes H_{\theta}^{d}(\mathrm{I})}, \quad\left(u \in \otimes_{i=1}^{n} \mathcal{W}_{i 1}\right),
\end{aligned}
$$

where $\mathcal{W}_{11}=H_{\max (0, \theta-m)}^{d}(\mathrm{I}) \cap H_{0, z_{p}}^{m}(\mathrm{I})$ and, for $i>1, \mathcal{W}_{i 1}=\tilde{H}_{\theta, z_{i}}^{d, m}(\mathrm{I})$. Corollary 2.8 and a tensor product argument show that

$$
\begin{aligned}
\left\|\otimes_{i=1}^{n}\left(P_{\ell_{i}}^{\beta, z_{i}}-P_{\ell_{i}-1}^{\beta, z_{i}}\right) u\right\|_{H^{m}(\mathrm{I}) \otimes L^{2}(\mathrm{I}) \otimes \cdots \otimes L^{2}(\mathrm{I})} & \\
& \lesssim 2^{-\left((d-m) \ell_{1}+d\left(\|\ell\|_{1}-\ell_{1}\right)\right)}\|u\|_{H_{\max (0, \theta-m)}^{d}(\mathrm{I}) \otimes H_{\theta}^{d}(\mathrm{I}) \otimes \cdots \otimes H_{\theta}^{d}(\mathrm{I})}, \quad\left(u \in \otimes_{i=1}^{n} \mathcal{W}_{i 1}\right) .
\end{aligned}
$$

Since $d-\nu(d-m)>0$, an application of Lemma 4.1 shows that

$$
\begin{aligned}
& \sum_{\left\{\ell: \ell_{1}+\nu\left(\|\ell\|_{1}-\ell_{1}\right)>L\right\}} 2^{-\left((d-m) \ell_{1}+d\left(\|\ell\|_{1}-\ell_{1}\right)\right)} \\
= & \sum_{j=L+1}^{\infty} \sum_{\left\{\ell: \ell_{1}+\nu\left(\|\ell\|_{1}-\ell_{1}\right) \in[j, j+1]\right\}} 2^{-(d-m)\left(\ell_{1}+\nu\left(\|\ell\|_{1}-\ell_{1}\right)\right)} 2^{-(d-\nu(d-m))\left(\|\ell\|_{1}-\ell_{1}\right)} \\
\lesssim & \sum_{j=L+1}^{\infty} 2^{-(d-m) j} \approx 2^{-(d-m) L}
\end{aligned}
$$

which completes the proof.

As in Theorem 4.3, let $\theta \in[0, d)$ and $\beta \in\left(\frac{\theta}{d}, 1\right)$. Then the combination of this theorem and Proposition 4.2 leads to the conclusion that

$$
\left\|u-\mathbf{P}_{L, 1}^{\beta} u\right\|_{L^{2}(\square)} \lesssim\left(\log \# \nabla_{L, 1}^{\beta}\right)^{(n-1)\left(\frac{1}{2}+d\right)}\left(\# \nabla_{L, 1}^{\beta}\right)^{-d}\|u\|_{\otimes_{i=1}^{n} H_{\theta}^{d}(\mathrm{I})}
$$

for all $u$ from the space in (4.1), and that for $\nu<\frac{d}{d-m}$,

$$
\left\|u-\mathbf{P}_{L, \nu}^{\beta} u\right\|_{H^{m}(\square)} \lesssim\left(\# \nabla_{L, \nu}^{\beta}\right)^{-(d-m)} \sqrt{\sum_{p=1}^{n}\|u\|_{\otimes_{i=1}^{n} H_{\theta-\delta_{p i} \min (m, \theta)}^{2}(\mathrm{I})}}
$$

for all $u \in \cap_{p=1}^{n} \otimes_{i=1}^{n} H_{\theta-\delta_{p i} \min (m, \theta)}^{d} \cap H_{0, \Gamma}^{m}(\square)$. To see the first estimate, note that with $N:=L^{n-1} 2^{L}$, we have $N^{\frac{1}{2}} \lesssim 2^{L} \leq N$, and so $L^{\frac{n-1}{2}} 2^{-d L}=L^{(n-1)\left(\frac{1}{2}+d\right)} N^{-d} \approx$ $(\log N)^{(n-1)\left(\frac{1}{2}+d\right)} N^{-d}$.

In the next section, we will demonstrate that for $u$ being the solution of the Poisson problem on $\square$ with homogeneous Dirichlet boundary conditions and a sufficiently smooth right-hand side, the norms of the right-hand side of both estimates are bounded. 


\section{Elliptic Regularity of LAPlace-Dirichlet PRoblem in ANisotropic WEIGHTED SOBOLEV SPACES}

In this section, we address the question of the regularity of the solution $u$ of Poisson's problem on $\square=(0,1)^{n}$ with homogeneous Dirichlet boundary conditions and sufficiently smooth right-hand side $f$ :

$$
u \in H_{0}^{1}(\square) \text { and }-\Delta u=f .
$$

Definition 5.1. For $i=1, \ldots, n$, let $\omega_{i}^{0}$ and $\omega_{i}^{1}$ be real numbers. We denote by $\underline{\omega}$ the collection $\left(\omega_{1}^{0}, \omega_{1}^{1}, \ldots, \omega_{n}^{1}\right)$. For $k \in \mathbb{N}_{0}$, we introduce the Hilbert space

$$
M_{\underline{\omega}}^{k}(\square)=\left\{u \in L_{\mathrm{loc}}^{2}(\square): \prod_{i=1}^{n}\left(x_{i}\right)^{\omega_{i}^{0}+\alpha_{i}}\left(1-x_{i}\right)^{\omega_{i}^{1}+\alpha_{i}} \partial_{x}^{\alpha} u \in L^{2}(\square), \forall \alpha,|\alpha| \leq k\right\}
$$

with its natural norm, denoted by $\|u\|_{M_{\underline{\underline{w}}}^{k}(\square)}$. In particular, $M_{\underline{\underline{0}}}^{k}(\square)$ denotes the space of $M_{\underline{\omega}}^{k}(\square)$ for which $\underline{\omega}=(0, \ldots, 0)$.

The main result of this section is the following:

Theorem 5.2. Let $\underline{\omega}$ be a collection $\left(\omega_{1}^{0}, \omega_{1}^{1}, \ldots, \omega_{n}^{1}\right)$ satisfying the conditions

$$
\omega_{i}^{0}, \omega_{i}^{1} \in\left(-\frac{3}{2}, 0\right), \forall i=1, \ldots, n, \quad \text { and } \sum_{i=1}^{n} \omega_{i}^{\delta_{i}}>-2, \forall \delta_{i} \in\{0,1\} .
$$

Let $k \geq 2$ be a natural number. If the right-hand side $f$ in (5.1) belongs to the space $M_{\underline{0}}^{k+2(n-2)}(\square)$, then the solution u of problem (5.1) belongs to $M_{\underline{\omega}}^{k}(\square)$ with the estimate

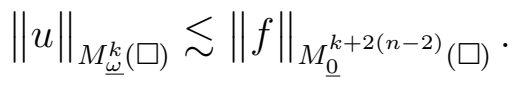

Remark 5.3. We assume that the dimension $n$ is $\geq 2$. Let $d \geq 1$ be an integer. Let $\theta<d$ be such that

If we choose $\underline{\omega}$ such that

$$
d-\theta<\frac{1}{n}
$$

$$
\omega_{1}^{\delta}=\theta-d-1 \quad \text { and } \quad \omega_{2}^{\delta}=\ldots=\omega_{n}^{\delta}=\theta-d,
$$

then $\underline{\omega}$ satisfies assumption (5.3), and we have the embedding

$$
M_{\underline{\omega}}^{d n}(\square) \subset H_{\theta-1}^{d}(\mathrm{I}) \otimes H_{\theta}^{d}(\mathrm{I}) \otimes \ldots \otimes H_{\theta}^{d}(\mathrm{I}),
$$

which proves that the solution of Poisson's problem on $\square$ with sufficiently smooth data satisfies the assumption of Theorem 4.3(b), and thus also that of Theorem 4.3(a).

Note that in the space on the left-hand side of (5.5) non-mixed partial derivatives $\partial_{i}^{k}$ up to order $d n$ are measured, whereas in the space at the right-hand side such partial derivatives are measured only up to order $d$.

The proof of Theorem 5.2 is organized in three steps:

(1) localization,

(2) basic regularity of the solution,

(3) successive estimates obtained by recurrence, each step of the recurrence using: 
(a) a dyadic partition argument,

(b) tangential regularity.

5.1. Localization. Let $\sigma \in\left[\frac{1}{2}, 1\right)$ be a real number. Let us denote by $\square_{\sigma}$ the cube $(0, \sigma)^{n}$. With obvious notations for functional spaces, by symmetry and covering, the estimate (5.4) will be proved if we show the following local estimate for a $\sigma^{\prime} \in(\sigma, 1)$ :

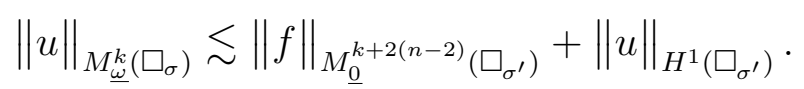

Note that on $\square_{\sigma}$ the weight $\left(1-x_{i}\right)$ is equivalent to 1 , so that

$$
M_{\underline{\omega}}^{k}\left(\square_{\sigma}\right)=\left\{u \in L_{\mathrm{loc}}^{2}\left(\square_{\sigma}\right): \prod_{i=1}^{n}\left(x_{i}\right)^{\omega_{i}^{0}+\alpha_{i}} \partial_{x}^{\alpha} u \in L^{2}\left(\square_{\sigma}\right), \forall \alpha,|\alpha| \leq k\right\}
$$

Thus we can denote $\omega_{i}^{0}$ by $\omega_{i}$.

The second localization is obtained by the introduction of an order between the coordinates: The cube is covered by $n$ ! domains isomorphic to

$$
\mathrm{T}=\left\{x \in \square: x_{1} \leq x_{2} \leq \ldots \leq x_{n}\right\} .
$$

For $\rho \in[1,2)$, let us denote by $\mathrm{T}_{\rho}$ the set

$$
\mathrm{T}_{\rho}=\left\{x \in \square_{\rho / 2}: x_{1} \leq \rho x_{2} \leq \ldots \leq \rho^{n-1} x_{n}\right\} .
$$

The estimate (5.6) will be proved if we show, for some $1 \leq \rho<\rho^{\prime}<2$ :

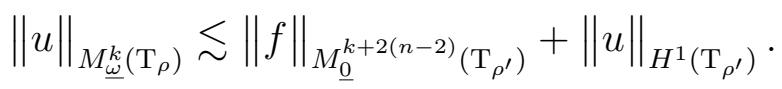

Finally, we note that we can assume the following conditions on the weight $\underline{\omega}$ :

$$
\omega_{1} \in\left(-\frac{3}{2},-1\right] \text { and } \omega_{i} \in(-1,0), i=2, \ldots, n \text { with } \omega_{1}+\ldots+\omega_{n}>-2
$$

Indeed, if we prove the estimate (5.9) with condition (5.10), we obviously prove the same estimate for all weights $\underline{\omega}^{\prime}$ such that

$$
\prod_{i=1}^{n}\left(x_{i}\right)^{\omega_{i}^{\prime}} \lesssim \prod_{i=1}^{n}\left(x_{i}\right)^{\omega_{i}} \quad \text { on } \quad \mathrm{T}_{\rho} .
$$

We can see that (5.11) holds for any pair of weights $\underline{\omega}, \underline{\omega}^{\prime}$ satisfying

$$
\omega_{1} \leq \omega_{1}^{\prime}, \quad \omega_{1}+\omega_{2} \leq \omega_{1}^{\prime}+\omega_{2}^{\prime}, \quad \ldots \quad \sum_{i=1}^{n} \omega_{i} \leq \sum_{i=1}^{n} \omega_{i}^{\prime}
$$

(estimate the quotient between the two members of (5.11)). Therefore for any weight $\underline{\omega}^{\prime}$ such that $\omega_{i}^{\prime} \in\left(-\frac{3}{2}, 0\right)$ with $\omega_{1}^{\prime}+\ldots+\omega_{n}^{\prime}>-2$, cf. conditions of Theorem 5.2 , it easy to find $\underline{\omega}$ satisfying (5.10) and (5.12), which proves the relevance of restricting to conditions (5.10) for the proof of Theorem 5.2. 


\subsection{Basic regularity of the solution.}

Lemma 5.4. The solution $u$ of problem (5.1) with a right-hand side $f \in L^{2}(\square)$, belongs to $H^{2}(\square)$ and satisfies the estimate

$$
\|u\|_{H^{2}(\square)} \lesssim\|f\|_{L^{2}(\square)}
$$

Proof. This regularity result is known to hold for any bounded convex domain [Gri85]. On the hypercube, it can be easily shown by a reflection argument: Odd reflections across the faces of the cube allow to define an extension $\tilde{u}$ of $u$ to a larger cube $\tilde{\square} \supset \square$, and such that $\Delta \tilde{u}=\tilde{f}$ where $\tilde{f}$ is the corresponding extension of $f$ and belongs to $L^{2}(\widetilde{\square})$. The ellipticity of $\Delta$ implies the $H^{2}$ regularity in $\square$.

Corollary 5.5. Let $V \subset V^{\prime} \subset \square$ be some open sets with $\square \cap \bar{V} \subset V^{\prime}$. Then for any $u \in H_{0}^{1}(\square)$ with $\Delta u \in L^{2}(\square)$, we have

$$
\|u\|_{H^{2}(V)} \lesssim\|\Delta u\|_{L_{2}\left(V^{\prime}\right)}+\|u\|_{H^{1}\left(V^{\prime}\right)} .
$$

Proof. Let $\chi$ be a smooth function with support in $V^{\prime}$ and equal to 1 on $V$. Then considering the formula

$$
\Delta(\chi u)=\chi \Delta u+2 \nabla \chi \cdot \nabla u+u \Delta \chi
$$

we deduce that

$$
\|\Delta(\chi u)\|_{L^{2}(\square)} \lesssim\|\Delta u\|_{L_{2}\left(V^{\prime}\right)}+\|u\|_{H^{1}\left(V^{\prime}\right)} .
$$

Since $\chi u$ obviously belongs to $H_{0}^{1}(\square)$, from Lemma 5.4 we deduce that $\chi u \in H^{2}(\square)$ with

$$
\|u\|_{H^{2}(V)} \leq\|\chi u\|_{H^{2}(\square)} \lesssim\|\Delta(\chi u)\|_{L^{2}(\square)} \lesssim\|\Delta u\|_{L^{2}\left(V^{\prime}\right)}+\|u\|_{H^{1}\left(V^{\prime}\right)}
$$

We also need some connections of $H^{2} \cap H_{0}^{1}(\square)$ with weighted spaces.

Lemma 5.6. Let $\omega_{1}, \ldots, \omega_{n} \in\left(-\frac{3}{2}, 0\right)$ such that $\omega_{1}+\ldots+\omega_{n}>-2$. Let $\rho \in(1,2)$. With the set $\mathrm{T}_{\rho}$ defined in (5.8), any $u \in H^{2} \cap H_{0}^{1}(\square)$ satisfies

$$
\left\|\left(\prod_{i=1}^{n} x_{i}^{\omega_{i}}\right) u\right\|_{L^{2}\left(\mathrm{~T}_{\rho}\right)}+\sum_{|\alpha|=1}\left\|\left(\prod_{i=1}^{n} x_{i}^{\omega_{i}}\right) x_{1} \partial_{x}^{\alpha} u\right\|_{L^{2}\left(\mathrm{~T}_{\rho}\right)} \lesssim\|u\|_{H^{2}(\square)} .
$$

Proof. Let us set $\bar{\omega}_{1}=\min \left\{\omega_{1}, \ldots, \omega_{n}\right\}$ and $\bar{\omega}_{2}=\omega_{1}+\ldots+\omega_{n}-\bar{\omega}_{1}$. On the set $\mathrm{T}_{\rho}$ there holds

$$
\prod_{i=1}^{n} x_{i}^{\omega_{i}} \lesssim x_{2}^{\bar{\omega}_{2}} x_{1}^{\bar{\omega}_{1}}
$$

(depending on $\rho$ ). Combining this with the tensor product properties of Sobolev spaces, it suffices to prove

$$
\left\|x_{2}^{\bar{\omega}_{2}} x_{1}^{\bar{\omega}_{1}} u\right\|_{L^{2}\left(\mathrm{~T}_{\rho}\right)}+\sum_{|\alpha|=1}\left\|x_{2}^{\bar{\omega}_{2}} x_{1}^{\bar{\omega}_{1}+1} \partial_{x}^{\alpha} u\right\|_{L^{2}\left(\mathrm{~T}_{\rho}\right)} \lesssim\|u\|_{H^{2}(\square)}
$$

for the dimension $n=2$. In other words we reduce to prove (5.13) in dimension $n=2$ for all $\omega_{1}>-\frac{3}{2}$ and all $\omega_{2} \geq \omega_{1}$ such that $\omega_{1}+\omega_{2}>-2$. 
For such a pair $\left(\omega_{1}, \omega_{2}\right)$, let $\varepsilon>0$ be such that

$$
\varepsilon-\frac{3}{2} \leq \omega_{1} \quad \text { and } \quad 2 \varepsilon-2 \leq \omega_{1}+\omega_{2}
$$

Using (5.11)-(5.12) we find $x_{2}^{\omega_{2}} x_{1}^{\omega_{1}} \lesssim x_{2}^{\varepsilon-\frac{1}{2}} x_{1}^{\varepsilon-\frac{3}{2}}$ on $\mathrm{T}_{\rho}$. Therefore what we want to prove is equivalent to showing that for all $\varepsilon>0$ and any $u \in H^{2} \cap H_{0}^{1}\left(\mathrm{I}^{2}\right)$

$$
\left\|x_{2}^{\varepsilon-\frac{1}{2}} x_{1}^{\varepsilon-\frac{3}{2}} u\right\|_{L^{2}\left(\mathrm{~T}_{\rho}\right)}+\sum_{|\alpha|=1}\left\|x_{2}^{\varepsilon-\frac{1}{2}} x_{1}^{\varepsilon-\frac{1}{2}} \partial_{x}^{\alpha} u\right\|_{L^{2}\left(\mathrm{~T}_{\rho}\right)} \lesssim\|u\|_{H^{2}\left(\mathrm{I}^{2}\right)} .
$$

The first step to prove (5.14) is to show that for any $v \in H^{1}\left(\mathrm{I}^{2}\right)$,

$$
\left\|x_{2}^{\varepsilon-\frac{1}{2}} x_{1}^{\varepsilon-\frac{1}{2}} v\right\|_{L^{2}\left(\mathrm{I}^{2}\right)} \lesssim\|v\|_{H^{1}\left(\mathrm{I}^{2}\right)} .
$$

This latter estimate relies on the combination of the one-dimensional estimate

$$
\left\|x^{-s} v\right\|_{L^{2}(\mathrm{I})} \lesssim\|v\|_{H^{s}(\mathrm{I})} \quad \text { for } \quad s<\frac{1}{2}
$$

(depending on $s$ ), which is a consequence of [Dau88, Th. AA.7], with the embedding $H^{1}\left(\mathrm{I}^{2}\right) \subset H^{s}(\mathrm{I}) \otimes H^{s}(\mathrm{I})$ for all $s \leq \frac{1}{2}$, which is a consequence of [Gri66, Th. 5.1].

Using (5.15) with $v=\partial_{x}^{\alpha} u$ for $|\alpha|=1$, we obtain

$$
\sum_{|\alpha|=1}\left\|x_{2}^{\varepsilon-\frac{1}{2}} x_{1}^{\varepsilon-\frac{1}{2}} \partial_{x}^{\alpha} u\right\|_{L^{2}\left(\mathrm{I}^{2}\right)} \lesssim\|u\|_{H^{2}\left(\mathrm{I}^{2}\right)} .
$$

Thanks to the fact that $u\left(0, x_{2}\right) \equiv 0$, the Hardy inequality with respect to the variable $x_{1}$ yields

$$
\left\|x_{2}^{\varepsilon-\frac{1}{2}} x_{1}^{\varepsilon-\frac{3}{2}} u\right\|_{L^{2}\left(\mathrm{I}^{2}\right)} \leq \frac{1}{1-\varepsilon}\left\|x_{2}^{\varepsilon-\frac{1}{2}} x_{1}^{\varepsilon-\frac{1}{2}} \partial_{x_{1}} u\right\|_{L^{2}\left(\mathrm{I}^{2}\right)} .
$$

Combining the last two estimates, we obtain (5.14) hence finally (5.13).

5.3. Weighted regularity of the solution. Our aim is to prove (5.9). As already mentioned, we can assume condition (5.10) on the weight $\underline{\omega}$. Let us choose $\rho \in[1,2)$ and $\delta \in\left(0, \frac{1}{8}\right)$, and set

$$
\mathrm{V}_{\ell}=\left\{x \in \mathrm{T}_{\rho}: \rho^{\ell} x_{\ell+1}>\delta\right\}, \quad \ell=0, \ldots, n-1, \quad \text { and } \quad \mathrm{V}_{n}=\mathrm{T}_{\rho} .
$$

For $2>\rho^{\prime}>\rho$ and $0<\delta^{\prime}<\delta$, we define similarly

$$
\mathrm{V}_{\ell}^{\prime}=\left\{x \in \mathrm{T}_{\rho^{\prime}}:\left(\rho^{\prime}\right)^{\ell} x_{\ell+1}>\delta^{\prime}\right\}, \quad \ell=0, \ldots, n-1, \quad \text { and } \quad \mathrm{V}_{n}=\mathrm{T}_{\rho^{\prime}},
$$

and also $\mathrm{V}_{\ell}^{\prime \prime}$ and $\mathrm{V}_{\ell}^{\prime \prime \prime}$ associated with some $2>\rho^{\prime \prime \prime}>\rho^{\prime \prime}>\rho^{\prime}$ and $0<\delta^{\prime \prime \prime}<\delta^{\prime \prime}<\delta^{\prime}$. We set $\omega_{i}:=\omega_{i}^{0}$.

The weight appearing in (5.7) is equivalent to a simpler weight on $\mathrm{V}_{\ell}$ :

$$
\prod_{i=1}^{n}\left(x_{i}\right)^{\omega_{i}+\alpha_{i}} \approx 1 \text { on } \mathrm{V}_{0} \text { and } \prod_{i=1}^{n}\left(x_{i}\right)^{\omega_{i}+\alpha_{i}} \approx \prod_{i=1}^{\ell}\left(x_{i}\right)^{\omega_{i}+\alpha_{i}} \quad \text { on } \mathrm{V}_{\ell}, \ell \geq 1 \text {. }
$$

In this section, we are going to prove by recurrence over $\ell=0, \ldots, n$ that

$$
\|u\|_{M_{\underline{\underline{\omega}}}^{k}\left(\mathrm{~V}_{\ell}\right)} \lesssim\|f\|_{M_{\underline{0}}^{k+2(\ell-1)}\left(\mathrm{V}_{\ell}^{\prime}\right)}+\|u\|_{H^{1}\left(\mathrm{~V}_{\ell}^{\prime}\right)},
$$

and finally deduce (5.9). 
- For $\ell=0$, the estimate (5.16) is simply

$$
\|u\|_{H^{k}\left(\mathrm{~V}_{0}\right)} \lesssim\|f\|_{H^{k-2}\left(\mathrm{~V}_{0}^{\prime}\right)}+\|u\|_{H^{1}\left(\mathrm{~V}_{0}^{\prime}\right)},
$$

and is a consequence of the ellipticity of the operator $\Delta$ and $V_{0} \subset \subset \square$.

- The proof of (5.16) for $\ell=1$ is done in two steps.

STEP 1. With the help of $\mathrm{V}_{0}$ we construct a locally finite dyadic covering of $\mathrm{V}_{1}$ : For a chosen $L>0, \mu \in \mathbb{N}_{0}$ and $\nu \in \mathbb{N}_{0}^{n-1}$ we denote by $\mathrm{V}_{1}^{\mu, \nu}$ the set

$$
\mathrm{V}_{1}^{\mu, \nu}=2^{-\mu}\left(\mathrm{V}_{0}+L(0, \nu)\right)=\left\{x=2^{-\mu}(y+L(0, \nu)), y \in \mathrm{V}_{0}\right\} .
$$

Likewise, we set

$$
\left(\mathrm{V}_{1}^{\prime}\right)^{\mu, \nu}=2^{-\mu}\left(\mathrm{V}_{0}^{\prime}+L(0, \nu)\right) .
$$

Then there exists $L>0$ and for each $\mu \in \mathbb{N}_{0}$ a subset $\mathbb{N}_{0, \mu}^{n-1}$ of $\mathbb{N}_{0}^{n-1}$ such that

$$
\mathrm{V}_{1} \subset \bigcup_{\mu \in \mathbb{N}_{0}} \bigcup_{\nu \in \mathbb{N}_{0, \mu}^{n-1}} \mathrm{~V}_{1}^{\mu, \nu} \subset \mathrm{V}_{1}^{\prime} \subset \bigcup_{\mu \in \mathbb{N}_{0}} \bigcup_{\nu \in \mathbb{N}_{0, \mu}^{n-1}}\left(\mathrm{~V}_{1}^{\prime}\right)^{\mu, \nu} \subset \mathrm{V}_{1}^{\prime \prime}
$$

Since $x_{1} \approx 1$ on $\mathrm{V}_{0}$, from the estimate (5.17) we deduce that for any $v \in H^{1}\left(\mathrm{~V}_{0}^{\prime}\right)$ such that $\Delta v \in H^{k-2}\left(\mathrm{~V}_{0}^{\prime}\right)$, there holds

$$
\sum_{|\alpha| \leq k}\left\|x_{1}^{\omega_{1}+|\alpha|} \partial_{x}^{\alpha} v\right\|_{\mathrm{V}_{0}} \lesssim \sum_{|\alpha| \leq k-2}\left\|x_{1}^{|\alpha|} \partial_{x}^{\alpha} \Delta v\right\|_{\mathrm{V}_{0}^{\prime}}+\sum_{|\alpha| \leq 1}\left\|x_{1}^{\omega_{1}+|\alpha|} \partial_{x}^{\alpha} v\right\|_{\mathrm{V}_{0}^{\prime}} .
$$

Here the norm $\|\cdot\|_{\mathrm{V}}$ means the $L^{2}$ norm on $\mathrm{V}$.

By the change of variables: $y \rightarrow x=2^{-\mu}(y+L(0, \nu))$ from $\mathrm{V}_{0}$ onto $\mathrm{V}_{1}^{\mu, \nu}$ we deduce from the previous estimate

$$
\begin{aligned}
& \sum_{|\alpha| \leq k} 2^{\mu \omega_{1}}\left\|x_{1}^{\omega_{1}+|\alpha|} \partial_{x}^{\alpha} u\right\|_{\mathrm{V}_{1}^{\mu, \nu}} \lesssim \\
& \sum_{|\alpha| \leq k-2} 2^{-2 \mu}\left\|x_{1}^{|\alpha|} \partial_{x}^{\alpha} f\right\|_{\left(\mathrm{V}_{1}^{\prime}\right)^{\mu, \nu}}+\sum_{|\alpha| \leq 1} 2^{\mu \omega_{1}}\left\|x_{1}^{\omega_{1}+|\alpha|} \partial_{x}^{\alpha} u\right\|_{\left(\mathrm{V}_{1}^{\prime}\right)^{\mu, \nu}}
\end{aligned}
$$

Since $2+\omega_{1} \geq 0$ and $\mu \geq 0$, this estimate yields:

$$
\sum_{|\alpha| \leq k}\left\|x_{1}^{\omega_{1}+|\alpha|} \partial_{x}^{\alpha} u\right\|_{\mathrm{V}_{1}^{\mu, \nu}} \lesssim \sum_{|\alpha| \leq k-2}\left\|x_{1}^{|\alpha|} \partial_{x}^{\alpha} f\right\|_{\left(\mathrm{V}_{1}^{\prime}\right)^{\mu, \nu}}+\sum_{|\alpha| \leq 1}\left\|x_{1}^{\omega_{1}+|\alpha|} \partial_{x}^{\alpha} u\right\|_{\left(\mathrm{V}_{1}^{\prime}\right)^{\mu, \nu}}
$$

Summing up the squares of the above estimates for all $\mu \in \mathbb{N}_{0}$ and $\nu \in \mathbb{N}_{0, \mu}^{n-1}$ we obtain

$$
\sum_{|\alpha| \leq k}\left\|x_{1}^{\omega_{1}+|\alpha|} \partial_{x}^{\alpha} u\right\|_{\mathrm{V}_{1}} \lesssim \sum_{|\alpha| \leq k-2}\left\|x_{1}^{|\alpha|} \partial_{x}^{\alpha} f\right\|_{\mathrm{V}_{1}^{\prime}}+\sum_{|\alpha| \leq 1}\left\|x_{1}^{\omega_{1}+|\alpha|} \partial_{x}^{\alpha} u\right\|_{\mathrm{V}_{1}^{\prime}} .
$$

To bound the the last term at the right hand side of this inequality, let $\chi$ be a smooth function with support in $\mathrm{V}_{1}^{\prime \prime}$ and equal to 1 in $\mathrm{V}_{1}^{\prime}$. There holds

$$
\sum_{|\alpha| \leq 1}\left\|x_{1}^{\omega_{1}+|\alpha|} \partial_{x}^{\alpha} u\right\|_{\mathrm{V}_{1}^{\prime}} \lesssim \sum_{|\alpha| \leq 1}\left\|x_{1}^{\omega_{1}+|\alpha|} \partial_{x}^{\alpha} \chi u\right\|_{\mathrm{V}_{1}^{\prime \prime}}
$$


Using that $\mathrm{V}_{1}^{\prime \prime} \subset \mathrm{T}_{\rho^{\prime \prime}}$, from Lemma 5.6 and Corollary 5.5 we deduce that

$$
\begin{aligned}
\sum_{|\alpha| \leq 1}\left\|x_{1}^{\omega_{1}+|\alpha|} \partial_{x}^{\alpha} \chi u\right\|_{\mathrm{T}_{\rho^{\prime \prime}}} & \lesssim\|\chi u\|_{H^{2}\left(\mathrm{~T}_{\rho^{\prime \prime}}\right)}=\|\chi u\|_{H^{2}\left(V_{1}^{\prime \prime}\right)} \\
& \lesssim\|\Delta(\chi u)\|_{V_{1}^{\prime \prime \prime}}+\|u\|_{H^{1}\left(V_{1}^{\prime \prime \prime}\right)} \lesssim\|f\|_{V_{1}^{\prime \prime \prime}}+\|u\|_{H^{1}\left(V_{1}^{\prime \prime \prime}\right)} .
\end{aligned}
$$

This estimate combined with estimate (5.19) gives

$$
\sum_{|\alpha| \leq k}\left\|x_{1}^{\omega_{1}+|\alpha|} \partial_{x}^{\alpha} u\right\|_{\mathrm{V}_{1}} \lesssim \sum_{|\alpha| \leq k-2}\left\|x_{1}^{|\alpha|} \partial_{x}^{\alpha} f\right\|_{\mathrm{V}_{1}^{\prime \prime \prime}}+\|u\|_{H^{1}\left(\mathrm{~V}_{1}^{\prime \prime \prime}\right)}
$$

Since the choice of the parameters $\rho^{\prime \prime \prime}$ and $\delta^{\prime \prime \prime}$ determining $V_{1}^{\prime \prime \prime}$ is arbitrary (the other parameters $\rho^{\prime \prime}, \rho^{\prime \prime}, \delta^{\prime}, \delta^{\prime \prime}$ and $L$ can be tuned accordingly), we may change notations in the above estimate, simply replacing $\mathrm{V}_{1}^{\prime \prime \prime}$ by $\mathrm{V}_{1}^{\prime}$ :

$$
\sum_{|\alpha| \leq k}\left\|x_{1}^{\omega_{1}+|\alpha|} \partial_{x}^{\alpha} u\right\|_{\mathrm{V}_{1}} \lesssim \sum_{|\alpha| \leq k-2}\left\|x_{1}^{|\alpha|} \partial_{x}^{\alpha} f\right\|_{\mathrm{V}_{1}^{\prime}}+\|u\|_{H^{1}\left(\mathrm{~V}_{1}^{\prime}\right)} .
$$

STEP 2. The aim of this step is to replace $|\alpha|$ by $\alpha_{1}$ in the left hand side of (5.20). This corresponds to improve the tangential regularity estimate.

Let $\mathbb{N}_{0} \ni q<k$. The estimate (5.20) implies in particular

$$
\sum_{0 \leq \alpha_{1} \leq q}\left\|x_{1}^{\omega_{1}+\alpha_{1}} \partial_{x_{1}}^{\alpha_{1}} u\right\|_{\mathrm{V}_{1}} \lesssim \sum_{|\alpha| \leq \max (q-2,0)}\left\|x_{1}^{\alpha_{1}} \partial_{x}^{\alpha} f\right\|_{\mathrm{V}_{1}^{\prime}}+\|u\|_{H^{1}\left(\mathrm{~V}_{1}^{\prime}\right)} .
$$

Let $\alpha_{2}^{\prime}=\left(\alpha_{2}, \ldots, \alpha_{n}\right)$ be a multi-index of length 1 . We denote $\left(x_{2}, \ldots, x_{n}\right)$ by $x_{2}^{\prime}$. Since

$$
\partial \mathrm{V}_{1}^{\prime} \cap \partial \square \subset\left\{x: x_{1}=0\right\}
$$

we can apply the above estimate to $\partial_{x_{2}^{\prime}}^{\alpha_{2}^{\prime}}$ and deduce

$$
\sum_{0 \leq \alpha_{1} \leq q}\left\|x_{1}^{\omega_{1}+\alpha_{1}} \partial_{x_{2}^{\prime}}^{\alpha_{2}^{\prime}} \partial_{x_{1}}^{\alpha_{1}} u\right\|_{\mathrm{V}_{1}} \lesssim \sum_{|\alpha| \leq \max (q-1,1)}\left\|x_{1}^{\alpha_{1}} \partial_{x}^{\alpha} f\right\|_{\mathrm{V}_{1}^{\prime}}+\|u\|_{H^{2}\left(\mathrm{~V}_{1}^{\prime}\right)}
$$

We combine with the bound $\|u\|_{H^{2}\left(\mathrm{~V}_{1}^{\prime}\right)} \lesssim\|f\|_{\mathrm{V}_{1}^{\prime \prime}}+\|u\|_{H^{1}\left(\mathrm{~V}_{1}^{\prime \prime}\right)}$ from Corollary 5.5 and obtain

$$
\sum_{0 \leq \alpha_{1} \leq q}\left\|x_{1}^{\omega_{1}+\alpha_{1}} \partial_{x_{2}^{\prime}}^{\alpha_{2}^{\prime}} \partial_{x_{1}}^{\alpha_{1}} u\right\|_{\mathrm{V}_{1}} \lesssim \sum_{|\alpha| \leq \max (q-1,1)}\left\|x_{1}^{\alpha_{1}} \partial_{x}^{\alpha} f\right\|_{\mathrm{V}_{1}^{\prime \prime}}+\|u\|_{H^{1}\left(\mathrm{~V}_{1}^{\prime \prime}\right)} .
$$

By the same considerations as above, we change $\mathrm{V}_{1}^{\prime \prime}$ to $\mathrm{V}_{1}^{\prime}$, and can proceed in the same way until $\left|\alpha_{2}^{\prime}\right|+q=k$, so that we arrive to

$$
\sum_{0 \leq \alpha_{1} \leq q}\left\|x_{1}^{\omega_{1}+\alpha_{1}} \partial_{x_{2}^{\prime}}^{\alpha_{2}^{\prime}} \partial_{x_{1}}^{\alpha_{1}} u\right\|_{\mathrm{V}_{1}} \lesssim \sum_{|\alpha| \leq \max (k-2, k-q)}\left\|x_{1}^{\alpha_{1}} \partial_{x}^{\alpha} f\right\|_{\mathrm{V}_{1}^{\prime \prime}}+\|u\|_{H^{1}\left(\mathrm{~V}_{1}^{\prime \prime}\right)} .
$$

Hence

$$
\sum_{|\alpha| \leq k}\left\|x_{1}^{\omega_{1}+\alpha_{1}} \partial_{x}^{\alpha} u\right\|_{\mathrm{V}_{1}} \lesssim \sum_{|\alpha| \leq k}\left\|x_{1}^{\alpha_{1}} \partial_{x}^{\alpha} f\right\|_{\mathrm{V}_{1}^{\prime}}+\|u\|_{H^{1}\left(\mathrm{~V}_{1}^{\prime}\right)}
$$


Finally, using condition (5.10) which ensures that $\omega_{1}+|\alpha| \leq 0$ for all $|\alpha| \leq 1$ we deduce from (5.21) the fully weighted estimate

$$
\sum_{|\alpha| \leq k}\left\|x_{1}^{\omega_{1}+\alpha_{1}} \partial_{x}^{\alpha} u\right\|_{\mathrm{V}_{1}} \lesssim \sum_{|\alpha| \leq k}\left\|x_{1}^{\alpha_{1}} \partial_{x}^{\alpha} f\right\|_{\mathrm{V}_{1}^{\prime}}+\sum_{|\alpha| \leq 1}\left\|x_{1}^{\omega_{1}+|\alpha|} \partial_{x}^{\alpha} u\right\|_{\mathrm{V}_{1}^{\prime}}
$$

- For $\ell=2$ we have the corresponding two steps: We incorporate the powers of $x_{2}$ in estimate (5.22) (which is allowed since $x_{2} \approx 1$ in $\mathrm{V}_{1}$ ), still denoting $\left(\alpha_{2}, \ldots, \alpha_{n}\right)$ by $\alpha_{2}^{\prime}$ :

$$
\sum_{|\alpha| \leq k}\left\|x_{2}^{\omega_{2}+\left|\alpha_{2}^{\prime}\right|} x_{1}^{\omega_{1}+\alpha_{1}} \partial_{x}^{\alpha} u\right\|_{\mathrm{V}_{1}} \lesssim \sum_{|\alpha| \leq k}\left\|x_{2}^{\left|\alpha_{2}^{\prime}\right|} x_{1}^{\alpha_{1}} \partial_{x}^{\alpha} f\right\|_{\mathrm{V}_{1}^{\prime}}+\sum_{|\alpha| \leq 1}\left\|x_{2}^{\omega_{2}} x_{1}^{\omega_{1}+|\alpha|} \partial_{x}^{\alpha} u\right\|_{\mathrm{V}_{1}^{\prime}} .
$$

We construct a dyadic covering of $\mathrm{V}_{2}$ with domains $\mathrm{V}_{2}^{\mu, \nu}$ similar to $\mathrm{V}_{1}$ (here $\nu$ belongs to $\mathbb{N}_{0}^{n-2}$ ). We use the fact that $2+\omega_{1}+\omega_{2}>0$ and obtain by similar steps as above

$$
\sum_{|\alpha| \leq k}\left\|x_{2}^{\omega_{2}+\left|\alpha_{2}^{\prime}\right|} x_{1}^{\omega_{1}+\alpha_{1}} \partial_{x}^{\alpha} u\right\|_{\mathrm{V}_{2}} \leq C\left\{\sum_{|\alpha| \leq k}\left\|x_{2}^{\left|\alpha_{2}^{\prime}\right|} x_{1}^{\alpha_{1}} \partial_{x}^{\alpha} f\right\|_{\mathrm{V}_{2}^{\prime}}+\|u\|_{H^{1}\left(\mathrm{~V}_{2}^{\prime}\right)}\right\} .
$$

We end the proof of (5.16) for $\ell=2$ using tangential derivatives $\partial_{x_{3}^{\prime}}^{\alpha_{3}^{\prime}}$, with $\alpha_{3}^{\prime}=$ $\left(\alpha_{3}, \ldots, \alpha_{n}\right)$ and $x_{3}^{\prime}=\left(x_{3}, \ldots, x_{n}\right)$.

- For $\ell=n$, only the first step is necessary, which, in the end, proves (5.16), hence (5.9).

5.4. Comments. There are not so many results about regularity in general polyhedra in any space dimension. Let us quote [MP77, MR91] for results in isotropic weighted spaces and [Dau88] for results in standard Sobolev spaces. Besides, for the space dimension $n \geq 3$, regularity in isotropic weighted spaces is by no means sufficient to ensure the assumptions of Theorem 4.3. The claim in [Nit05] that in dimension $n=3$, a regularity result in isotropic weighted spaces from [MR91] would guarantee the assumptions of Theorem 4.3 seems to be caused by a mistake in the reproduction of the definition of [MR91]'s weighted spaces.

The spaces which we consider in our paper are anisotropic, requiring more regularity in directions tangential to edges of any dimensions. Let us quote the note [BCD03] where similar spaces are employed on three-dimensional polyhedra. Thus, to our knowledge, Theorem 5.2 is new.

With suitable definitions for the weighted spaces $M_{\underline{\omega}}^{k}$ (compare with [BCD03] in dimension $n=3$ ) our result on the regularity of solution of Poisson's problem (5.1) can be extended to any $n$-dimensional polyhedron $\Omega$ along the following lines:

- If the polyhedron $\Omega$ is convex, the $H^{2}$ regularity (Lemma 5.4) still holds, and Theorem 5.2 is true with the same conditions on $\underline{\omega}$.

- If the polyhedron $\Omega$ is not convex, the solution $u$ with a right-hand side $f \in L^{2}(\Omega)$ does not belong to $H^{2}(\Omega)$ in general, but there exists $s=s_{\Omega}>1$ so that $u \in H^{s}(\Omega)$, see [Dau88]. Then it could be proved that the conclusion 
of Theorem 5.2 holds under the stronger condition on $\underline{\omega}$ :

$$
\omega_{i}^{0}, \omega_{i}^{1} \in\left(-\frac{3}{2}, 0\right), \forall i=1, \ldots, n, \quad \text { and } \quad \sum_{i=1}^{n} \omega_{i}^{\delta_{i}}>-s, \forall \delta_{i} \in\{0,1\} .
$$

Likewise, Theorem 5.2 could be generalized to the Dirichlet problem in $H_{0}^{m}$ associated with any coercive sesquilinear form of order $m$ (operator of order $2 m$ ) with smooth coefficients.

In contrast, the solution $u$ of the Neumann problem for the Laplace operator on $\square$ does not belong to $M_{\omega}^{k}(\square)$ under condition (5.3) in general. Nevertheless, if conditions appearing in (5.2) are restricted to the case when $\alpha_{1}, \ldots \alpha_{n} \geq 1$, we can still hope that $u$ fulfills such conditions.

\section{The approximation of the solution of Poisson's Problem using STANDARD SPARSE GRIDS}

Without local refinement, i.e., $\beta=0$, the estimates from Theorem 2.6 read as

$$
\begin{aligned}
\left\|u-P_{\ell}^{0} u\right\|_{L^{2}(\mathrm{I})} & \lesssim 2^{-d \ell}\|u\|_{H^{d}(\mathrm{I})} \quad\left(u \in H^{d}(\mathrm{I}) \cap H_{0, z}^{m}(\mathrm{I})\right) \\
\left\|u-P_{\ell}^{0} u\right\|_{H^{m}(\mathrm{I})} & \lesssim 2^{-(d-m) \ell}\|u\|_{H^{d}(\mathrm{I})} \quad\left(u \in H^{d}(\mathrm{I}) \cap H_{0, z}^{m}(\mathrm{I})\right) .
\end{aligned}
$$

More generally, with the interpolation space $\mathcal{H}_{0, z}^{t}(\mathrm{I}):=\left[L^{2}(\mathrm{I}), H^{d}(\mathrm{I}) \cap H_{0, z}^{m}(\mathrm{I})\right]_{\frac{t}{d}, 2}$, it holds that

$$
\begin{aligned}
\left\|u-P_{\ell}^{0} u\right\|_{L^{2}(\mathrm{I})} & \lesssim 2^{-t \ell}\|u\|_{\mathcal{H}_{0, z}^{t}(\mathrm{I})} \quad\left(t \in[0, d], u \in \mathcal{H}_{0, z}^{t}(\mathrm{I})\right), \\
\left\|u-P_{\ell}^{0} u\right\|_{H^{m}(\mathrm{I})} & \lesssim 2^{-(t-m) \ell}\|u\|_{\mathcal{H}_{0, z}^{t}(\mathrm{I})} \quad\left(t \in[m, d], u \in \mathcal{H}_{0, z}^{t}(\mathrm{I})\right) .
\end{aligned}
$$

Similarly to Theorem 4.3(a), we arrive at

$$
\left\|u-\mathbf{P}_{L, 1}^{0} u\right\|_{L^{2}(\square)} \lesssim L^{\frac{n-1}{2}} 2^{-s L}\|u\|_{\otimes_{i=1}^{n} \mathcal{H}_{0, z_{i}}^{s}(\mathrm{I})}\left(s \in[0, d], u \in \otimes_{i=1}^{n} \mathcal{H}_{0, z_{i}}^{s}(\mathrm{I})\right)
$$

By applying (6.1) with $t=s$, and (6.2) with $t=s-m+\varepsilon$, similarly to Theorem 4.3(b), we infer that for $s \in[m, d], \varepsilon \in(0, m]$ and $(s-m) \nu<(s-m)+\varepsilon$,

$$
\left\|u-\mathbf{P}_{L, \nu}^{0} u\right\|_{H^{m}(\square)} \lesssim 2^{-(s-m) L} \sqrt{\sum_{p=1}^{n}\|u\|_{\otimes_{i=1}^{n} \mathcal{H}_{0, z_{i}}^{s+\left(1-\delta_{p i}\right)(\varepsilon-m)}(\mathrm{I})}^{2}}
$$

for all $u \in \cap_{p=1}^{n} \otimes_{i=1}^{n} \mathcal{H}_{0, z_{i}}^{s+\left(1-\delta_{p i}\right)(\varepsilon-m)}(\mathrm{I})$, where, by taking $\nu>1,2^{-(s-m) L} \approx\left(\# \boldsymbol{\nabla}_{L, \nu}^{0}\right)^{-(s-m)}$.

The estimates (6.1), (6.2) and, similarly, (6.3) are sharp in the sense that, generally, a decay of the error like $2^{-t \ell}, 2^{-(t-m) \ell}$ or $2^{-(s-m) L}$ is not valid when only $u \in \mathcal{H}_{0, z_{i}}^{t^{\prime}}(\mathrm{I})$ for some $t^{\prime}<t$, or $u \in \cap_{p=1}^{n} \otimes_{i=1}^{n} \mathcal{H}_{0, z_{i}}^{s^{\prime}+\left(1-\delta_{p i}\right)\left(\varepsilon^{\prime}-m\right)}(\mathrm{I})$ for some $s^{\prime}<s$ or $\varepsilon^{\prime} \leq 0$. Note that we had to include the word "generally" in the preceding sentence, because higher order convergence for less smooth $u$ does hold when $u$ happens to be a finite linear combination of wavelets $\boldsymbol{\psi}_{\boldsymbol{\lambda}}$, or when it is exceptionally close to such a function. 
In the following, we again consider $u$ to be the solution of Poisson's problem on $\square=(0,1)^{n}$ with homogeneous Dirichlet boundary conditions and sufficiently smooth right-hand side $f$ :

$$
u \in H_{0}^{1}(\square) \text { and }-\Delta u=f,
$$

i.e., $m=1$ and $z_{i}=\{0,1\}$. We will show that for sufficiently smooth right-hand sides $f$ and $n \geq 2$, for

$$
s \in\left[1, \frac{3}{2}+\frac{1}{n}\right) \Longrightarrow u \in \cap_{p=1}^{n} \otimes_{i=1}^{n} \mathcal{H}_{0,\{0,1\}}^{s-1+\delta_{i p}}(\mathrm{I}),
$$

whilst for $s>\frac{3}{2}+\frac{1}{n}$, generally $u$ is not in that space (it will be when additionally $f$ vanishes at a sufficiently high order at the boundary). In view of the estimate (6.3), and the discussion following it, these results mean that the error, measured in $H^{1}(\square)$, in the best approximation from the optimized sparse-grid space of dimension $N$ of the solution of Poisson's problem on the $n$-dimensional hypercube decays like $N^{-(s-1)}$ for any $s<\frac{3}{2}+\frac{1}{n}$, whilst, generally, this estimate does not hold for $s>\frac{3}{2}+\frac{1}{n}$. Note that here we could write "best approximation", since the error $u-\mathbf{P}_{L, \nu}^{0} u$, measured in $\|\cdot\|_{H^{1}(\square)}$ is, indeed, up to a constant factor, equal to the error in the best approximation from $\operatorname{span} \mathbf{P}_{L, \nu}^{0}$, because the projector $\mathbf{P}_{L, \nu}^{0}$ is uniformly bounded in $H^{1}(\square)$-norm. The reason is that the wavelet basis $\left\{\left(\sum_{i=1}^{n} 4^{\left|\lambda_{i}\right|}\right)^{-1 / 2} \boldsymbol{\psi}_{\boldsymbol{\lambda}}: \boldsymbol{\lambda} \in \boldsymbol{\nabla}\right\}$ is a Riesz basis for $H_{0, \Gamma}^{1}(\square)$.

In a way the above result is favourable. Indeed, unlike with standard "full grid" approximation, the rate $\frac{1}{2}+\frac{1}{n}$, although mildly dependent on $n$, does not decrease to 0 when $n \rightarrow \infty$. On the other hand, however, the rate $\frac{1}{2}+\frac{1}{n}$ does not show any benefit of taking piecewise polynomial wavelets of orders $d$ larger than 2 , and depending on the value of $d$, this rate is (much) worse than the optimal rate $d-1$ that is realized with the approach of combining optimized sparse grids with local refinements outlined in the preceding sections.

Before continuing, we note that for $s \geq 1, s \neq \frac{3}{2}$,

$$
\cap_{p=1}^{n} \otimes_{i=1}^{n} \mathcal{H}_{0,\{0,1\}}^{s-1+\delta_{i p}}(\mathrm{I})=\cap_{p=1}^{n} \otimes_{i=1}^{n} H^{s-1+\delta_{i p}}(\mathrm{I}) \cap H_{0}^{1}(\square) .
$$

Indeed, since

$$
\mathcal{H}_{0,\{0,1\}}^{t}(\mathrm{I})= \begin{cases}H^{t}(\mathrm{I}) & t \in\left[0, \frac{1}{2}\right), \\ \left\{u \in H^{t}(I): \gamma_{v} u=0, v \in\{0,1\}\right\} & t>\frac{1}{2},\end{cases}
$$

the proof of (6.6) follows that of $(3.4 \mathrm{a})$, for $t \in\left[0, \frac{1}{2}\right)$ taking the first first option in (3.4c), and for $t>\frac{1}{2}$ taking the second option. Since we know that the solution of Poisson's problem is in $H_{0}^{1}(\square)$, what is left is to show that for

$$
s \in\left[1, \frac{3}{2}+\frac{1}{n}\right) \Longrightarrow \partial_{p} u \in \otimes_{i=1}^{n} H^{s-1}(\mathrm{I}) \quad(1 \leq p \leq n),
$$

known as a Sobolev space of dominating mixed smoothness, and that this is generally not true for $s>\frac{3}{2}+\frac{1}{n}$.

In order to do so, we apply a Fourier analysis. For $k \in \mathbb{N}$, let $\phi_{k}(x):=\sqrt{2} \sin (k \pi x)$, $\psi_{k}(x)=\sqrt{2} \cos (k \pi x)$, and let $\psi_{0}=1$. It is clear that $\left\{\phi_{k}: k \in \mathbb{N}\right\}$ is the normed eigenvector basis of the self-adjoint operator $\varphi \mapsto-\varphi^{\prime \prime}$ with domain $H^{2} \cap H_{0}^{1}(\mathrm{I})$ 
(Dirichlet). Likewise, $\left\{\psi_{k}: k \in \mathbb{N}_{0}\right\}$ is the normed eigenvector basis of the selfadjoint operator $\varphi \mapsto-\varphi^{\prime \prime}$ with domain $\left\{\varphi \in H^{2}(\mathrm{I}), \varphi^{\prime}(0)=\varphi^{\prime}(1)=0\right\}$ (Neumann). Furthermore $\phi_{k}^{\prime}=k \pi \psi_{k}$ and $\psi_{k}^{\prime}=-k \pi \phi_{k}$. As a consequence we obtain immediately.

Lemma 6.1. $\left\{\phi_{k}: k \in \mathbb{N}\right\}$ and $\left\{\psi_{k}: k \in \mathbb{N}_{0}\right\}$ are orthonormal bases for $L^{2}(\mathrm{I})$.

Lemma 6.2. $\left\{\frac{\phi_{k}}{k \pi}: k \in \mathbb{N}\right\}\left(\left\{\frac{\phi_{k}}{\sqrt{1+k^{2} \pi^{2}}}: k \in \mathbb{N}\right\}\right)$ is an orthonormal basis of $H_{0}^{1}(\mathrm{I})$ equipped with $|\cdot|_{H^{1}(\mathrm{I})}\left(\|\cdot\|_{H^{1}(\mathrm{I})}\right)$, and $\left\{\frac{\psi_{k}}{\sqrt{1+k^{2} \pi^{2}}}: k \in \mathbb{N}_{0}\right\}$ is an orthonormal basis of $H^{1}(\mathrm{I})$.

By taking tensor products, we conclude that

$$
\left\{\phi_{\mathbf{k}}:=\otimes_{i=1}^{n} \phi_{k_{i}}: \mathbf{k} \in \mathbb{N}^{n}\right\}, \quad\left\{\frac{\phi_{\mathbf{k}}}{\pi\|\mathbf{k}\|_{2}}: \mathbf{k} \in \mathbb{N}^{n}\right\}
$$

are orthonormal bases for

$$
L^{2}(\square), \quad H_{0}^{1}(\square)
$$

respectively, the latter space being equipped with $|\cdot|_{H^{1}(\square)}:=\sqrt{\sum_{i=1}^{n}\left\|\partial_{i} \cdot\right\|_{L^{2}(\square)}^{2}}$.

By using this basis, the stiffness matrix resulting from the variational formulation of the Poisson problem is the identity, and we conclude that for a right-hand side $f \in\left(H_{0}^{1}(\square)\right)^{\prime}$, the solution $u$ is given by

$$
u=\sum_{\mathbf{k} \in \mathbb{N}^{n}} f\left(\frac{\phi_{\mathbf{k}}}{\pi\|\mathbf{k}\|_{2}}\right) \frac{\phi_{\mathbf{k}}}{\pi\|\mathbf{k}\|_{2}}
$$

and so

$$
\partial_{p} u(\boldsymbol{x})=\sum_{\mathbf{k} \in \mathbb{N}^{n}} \frac{k_{p}}{\|\mathbf{k}\|_{2}} f\left(\frac{\phi_{\mathbf{k}}}{\pi\|\mathbf{k}\|_{2}}\right) \psi_{k_{p}}\left(x_{p}\right) \prod_{i \neq p} \phi_{k_{i}}\left(x_{i}\right), \quad(1 \leq p \leq n) .
$$

To estimate the decay of the coefficients in this expansion for $\|\mathbf{k}\|_{2} \rightarrow \infty$, we note that for $g \in W^{1,1}(\mathrm{I})\left(\hookrightarrow L^{\infty}(\mathrm{I})\right)$,

$$
\int_{\mathrm{I}} g(x) \phi_{k}(x) d x=-\left.g(x) \frac{\psi_{k}(x)}{k \pi}\right|_{0} ^{1}+\int_{\mathrm{I}} g^{\prime}(x) \frac{\psi_{k}(x)}{k \pi} d x,
$$

and so $\left|\int_{\mathrm{I}} g(x) \phi_{k}(x) d x\right| \lesssim 1 / k$, where a decay of higher order can only be expected when $g$ vanishes at $\partial \mathrm{I}$ (see Remark 6.4). As a consequence, if $f \in \otimes_{i=1}^{n} W^{1,1}(\mathrm{I})$ $\left(\hookrightarrow L^{\infty}(\mathrm{I})\right)$, then

$$
\left|f\left(\frac{\phi_{\mathbf{k}}}{\pi\|\mathbf{k}\|_{2}}\right)\right| \lesssim\left(\|\mathbf{k}\|_{2} \prod_{i=1}^{n} k_{i}\right)^{-1}
$$

which for $f$ that do not vanish at $\partial \square$ is generally sharp. For example, for $f=1$,

$$
f\left(\frac{\phi_{\mathbf{k}}}{\pi\|\mathbf{k}\|_{2}}\right)=\left\{\begin{array}{cl}
\frac{1}{\pi\|\mathbf{k}\|_{2}}\left(\frac{2 \sqrt{2}}{\pi}\right)^{n} \frac{1}{\prod_{i=1}^{n} k_{i}} & \text { when } \mathbf{k} \in(2 \mathbb{N}+1)^{n}, \\
0 & \text { otherwise. }
\end{array}\right.
$$


Lemma 6.3. For $v=\sum_{\mathbf{k} \in \prod_{i=1}^{n} \mathbb{K}_{i}} c_{\mathbf{k}} v_{k_{1}}^{(1)} \otimes \cdots \otimes v_{k_{n}}^{(n)}$, where $\mathbb{K}_{p}=\mathbb{N}_{0}$ and $v_{k_{p}}^{(p)}=\psi_{k_{p}}$, and $\mathbb{K}_{i}=\mathbb{N}$ and $v_{k_{i}}^{(i)}=\phi_{k_{i}}$ for $i \neq p$, for $t \in[0,1] \backslash\left\{\frac{1}{2}\right\}$ we have

$$
\|v\|_{H^{t}(\mathrm{I}) \otimes \cdots \otimes H^{t}(\mathrm{I})} \approx\left\|\mathbf{k} \mapsto\left(\prod_{i=1}^{n} \sqrt{1+k_{i}^{2} \pi^{2}}\right)^{t} c_{\mathbf{k}}\right\|_{\ell^{2}\left(\prod_{i=1}^{n} \mathbb{K}_{i}\right)}
$$

Proof. For any $\mathbf{k} \in \prod_{i=1}^{n} \mathbb{K}_{i}$, let $w_{\mathbf{k}}:=\prod_{i=1}^{n} \sqrt{1+k_{i}^{2} \pi^{2}}$, and

$$
\ell_{w}^{2}\left(\prod_{i=1}^{n} \mathbb{K}_{i}\right):=\left\{c \in \ell^{2}\left(\prod_{i=1}^{n} \mathbb{K}_{i}\right):\|c\|_{\ell_{w}^{2}\left(\prod_{i=1}^{n} \mathbb{K}_{i}\right)}:=\sqrt{\sum_{\mathbf{k} \in \prod_{i=1}^{n} \mathbb{K}_{i}}\left|w_{\mathbf{k}} c_{\mathbf{k}}\right|^{2}}<\infty\right\},
$$

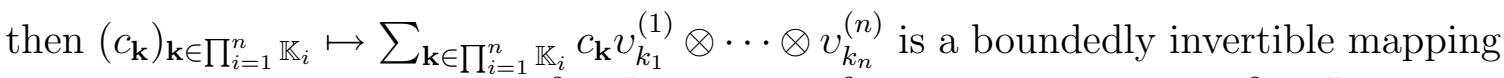
(even an isomorphism) from $\ell^{2}\left(\prod_{i=1}^{n} \mathbb{K}_{i}\right) \rightarrow L^{2}(\square)$ as well as from $\ell_{w}^{2}\left(\prod_{i=1}^{n} \mathbb{K}_{i}\right) \rightarrow$ $\mathcal{H}^{1}(\square):=H_{0}^{1}(\mathrm{I}) \otimes \cdots \otimes H^{1}(\mathrm{I}) \otimes \cdots \otimes H_{0}^{1}(\mathrm{I})$, with the space $H^{1}(\mathrm{I})$ being on the $p$ th position. By definition of an interpolation space, for $t \in[0,1]$, the mapping is also boundedly invertible from $\ell_{w^{t}}^{2}\left(\prod_{i=1}^{n} \mathbb{K}_{i}\right) \rightarrow\left[L_{2}(\square), \mathcal{H}^{1}(\square)\right]_{t, 2}=\left[L_{2}(\mathrm{I}), H_{0}^{1}(\mathrm{I})\right]_{t, 2} \otimes \cdots \otimes$ $\left[L_{2}(\mathrm{I}), H^{1}(\mathrm{I})\right]_{t, 2} \otimes \cdots \otimes\left[L_{2}(\mathrm{I}), H_{0}^{1}(\mathrm{I})\right]_{t, 2}$. Since $\left[L_{2}(\mathrm{I}), H^{1}(\mathrm{I})\right]_{t, 2}=H^{t}(\mathrm{I}),\left[L_{2}(\mathrm{I}), H_{0}^{1}(\mathrm{I})\right]_{t, 2}=$ $H_{0}^{t}(\mathrm{I})$ when $t>\frac{1}{2}$, and $\left[L_{2}(\mathrm{I}), H_{0}^{1}(\mathrm{I})\right]_{t, 2}=H^{t}(\mathrm{I})$ when $t<\frac{1}{2}$, in all cases with a norm that is equivalent to the $H^{t}(\mathrm{I})$-norm, the proof is completed.

The estimate (6.9) is equivalent to

$$
\left|\left(\prod_{i=1}^{n} \sqrt{1+\pi^{2} k_{i}^{2}}\right)^{s-1} \frac{k_{p}}{\|\mathbf{k}\|_{2}} f\left(\frac{\phi_{\mathbf{k}}}{\pi\|\mathbf{k}\|_{2}}\right)\right| \lesssim \frac{k_{p}}{\|\mathbf{k}\|_{2}^{2}}\left(\prod_{i=1}^{n} k_{i}\right)^{s-2} .
$$

We claim that the right-hand side is in $\ell^{2}\left(\mathbb{N}^{n}\right)$ iff $s-1<\frac{1}{2}+\frac{1}{n}$. Indeed,

$$
\frac{k_{p}}{\|\mathbf{k}\|_{2}^{2}}\left(\prod_{i=1}^{n} k_{i}\right)^{s-2} \leq \frac{1}{\|\mathbf{k}\|_{2}}\left(\prod_{i=1}^{n} k_{i}\right)^{s-2} \leq\left(\prod_{i=1}^{n} k_{i}\right)^{s-2-\frac{1}{n}}
$$

and the right-hand side is in $\ell^{2}\left(\mathbb{N}^{n}\right)$ iff $2\left(s-2-\frac{1}{n}\right)<-1$ or $s-1<\frac{1}{2}+\frac{1}{n}$. Up to a constant factor, that may depend on $n$, the estimates from (6.10) are sharp for all $\mathbf{k} \in$ $\mathbb{N}^{n}$ with, say, $\max _{i} k_{i} \leq 2 \min _{i} k_{i}$, whereas for those $\mathbf{k},\left(\prod_{i=1}^{n} k_{i}\right)^{s-2-\frac{1}{n}} \approx\|\mathbf{k}\|_{2}^{(s-2) n-1}$. Clearly, $\sum_{\left\{\mathbf{k} \in \mathbb{N}^{n}: \max _{i} k_{i} \leq 2 \min _{i} k_{i}\right\}}\|\mathbf{k}\|_{2}^{((s-2) n-1) 2}<\infty$ iff $\sum_{\mathbf{k} \in \mathbb{N}^{n}}\|\mathbf{k}\|_{2}^{((s-2) n-1) 2}<\infty$, the latter being the case iff $2(s-2) n-2+(n-1)<-1$ or $s-1<\frac{1}{2}+\frac{1}{n}$, which proves our claim.

Since $\frac{1}{2}+\frac{1}{n} \leq 1$ when $n \geq 2$, this last result in combination with (6.8), (6.9) and Lemma 6.3 proves our earlier claim about the regularity in Sobolev spaces of dominating mixed smoothness of the (first order partial derivatives of the) solution of Poisson's problem on the $n$-dimensional hypercube, and with that our claim about the rate of convergence of the optimized sparse-grid approximation without local refinement.

Remark 6.4. If $f \in \otimes_{i=1}^{n} W_{0}^{d-1,1}(\mathrm{I}) \cap W^{d, 1}(\mathrm{I})=\left\{g \in \otimes_{i=1}^{n} W^{d, 1}(\mathrm{I}): \partial_{n}^{r} g=0\right.$ on $\partial \square$, $0 \leq r \leq d-2\}$, then repeated integration by parts shows that (6.9) can be replaced 
by

$$
\left|f\left(\frac{\phi_{\mathbf{k}}}{\pi\|\mathbf{k}\|_{2}}\right)\right| \lesssim\left(\|\mathbf{k}\|_{2} \prod_{i=1}^{n} k_{i}^{d}\right)^{-1} .
$$

Then for $s<d+\frac{1}{2}+\frac{1}{n}$ one shows that $\partial_{p} u \in \otimes_{i=1}^{n} H^{s-1}(\mathrm{I})(1 \leq p \leq n)$, meaning that for $\nu \in\left(1,1+\frac{1 / 2+1 / n}{d-1}\right),\left\|u-\mathbf{P}_{L, \nu}^{0} u\right\|_{H^{1}(\square)} \lesssim\left(\# \nabla_{L, \nu}^{0}\right)^{-(d-1)}$. So if the right-hand $f$ is sufficiently smooth and vanishes at the boundary at a sufficiently high order, then already the optimized sparse-grid approximation without local refinement of the solution of Poisson's problem converges in $H^{1}(\square)$-norm with the best possible, dimension independent rate.

Remark 6.5. Because of the explicit expression (6.7), one may think of approximating $u$ by the expansion formed by its $N$ largest Fourier coefficients. Assuming a general smooth right-hand side $f$, based on (6.9) one may verify (cf. [Dij09]) that the error measured in $H^{1}(\square)$ of such an approximation with $N$ coefficients is generally not better than of order $N^{-\left(\frac{1}{2}+\frac{1}{n}\right)}$, i.e., the approximation rate is equal to that of the optimized sparse-grid approximation without local refinement. The computational complexity of computing the $N$ largest Fourier coefficients is much higher though.

Remark 6.6. The bound $\frac{3}{2}+\frac{1}{n}$ in (6.5) is due to the presence of a singular part in the solutions of the Dirichlet problem (6.4). When $n=2$, as is well known [Gri85, Dau88], the first contribution to this singular part attached to the corner $(0,0)$ is the function

$$
\left(x_{1}, x_{2}\right) \mapsto f(0,0)\left\{\frac{1}{\pi} r^{2}(\log r \sin 2 \theta+\theta \cos 2 \theta)+\frac{1}{2} x_{2}^{2}\right\},
$$

with the polar coordinate $(r, \theta)$. Note the coefficient $f(0,0)$. Taking similar contributions at four corners into account, we see that if $f$ is not zero at one corner, then $u \notin H^{3}(\square)$. In contrast, if $f$ is zero at all corners, $u \in H^{5-\varepsilon}(\square)$ for all $\varepsilon>0$, because the next singularity is in $r^{4} \log r$. More generally, if $f$ and all its derivatives are zero at all corners, then $u \in H^{d}(\square)$ for all $d \in \mathbb{N}$. In fact, the necessary and sufficient condition for the infinite smoothness of $u$ up to corners is even weaker: it requires that at four corners $\mathbf{c}_{i}, i=1,2,3,4, f\left(\mathbf{c}_{i}\right)=0,\left(\partial_{1}^{2}-\partial_{2}^{2}\right) f\left(\mathbf{c}_{i}\right)=0$, and in general $A_{i}^{2 j} f\left(\mathbf{c}_{i}\right)=0$, where $A_{i}^{2 j}$ are operators of order $2 j, j \in \mathbb{N}_{0}$.

In higher dimensions $n$, the singular part is still related to the traces of $f$ on the edges of $\square$. This feature is very particular to the Laplacian and to the domain $\square$ : It is related to the validity of the reflection principle, cf. the proof of Lemma 5.4 - for a smooth right hand side $f$ with compact support in $\square$, this principle yields immediately the infinite smoothness of the solution $u$.

When $n=3$, the solutions of the Dirichlet problem (6.4) in more general polyhedral domains than $\square$ have singular parts which involve distance functions to the singular sets of the domain - edges and corners - and not the distance to the faces of the domain, see [Dau88, Ch.17]. But, unless all opening angles have the form $\frac{\pi}{\ell}$ with integers $\ell$, the fact that $f \equiv 0$ on the boundary does not improve the regularity of the solution in general.

\section{REFERENCES}

[Ada75] R.A. Adams. Sobolev Spaces. Academic Press, New York, 1975. 
[BCD03] A. Buffa, M. Costabel, and M. Dauge. Anisotropic regularity results for Laplace and Maxwell operators in a polyhedron. C. R. Acad. Sc. Paris, Série I, 336:565-570, 2003.

[BG04] H.J. Bungartz and M. Griebel. Sparse grids. Acta Numer., 13:147-269, 2004.

[CDV93] A. Cohen, I. Daubechies, and P. Vial. Wavelets on the interval and fast wavelet transforms. Appl. Comput. Harmon. Anal., 1(1):54-81, 1993.

[CZ52] A. P. Calderon and A. Zygmund. On the existence of certain singular integrals. Acta Math., 88:85-139, 1952.

[Dah96] W. Dahmen. Stability of multiscale transformations. J. Fourier Anal. Appl., 4:341-362, 1996.

[Dau88] M. Dauge. Elliptic Boundary Value Problems in Corner Domains - Smoothness and Asymptotics of Solutions. Lecture Notes in Mathematics, Vol. 1341. Springer-Verlag, Berlin, 1988.

[Dij09] T.J. Dijkema. Adaptive tensor product wavelet methods for solving PDEs. PhD thesis, Utrecht University, 2009.

[DKT98] R. A. DeVore, S. V. Konyagin, and V. N. Temlyakov. Hyperbolic wavelet approximation. Constr. Approx., 14(1):1-26, 1998.

[DKU99] W. Dahmen, A. Kunoth, and K. Urban. Biorthogonal spline-wavelets on the interval Stability and moment conditions. Appl. Comp. Harm. Anal., 6:132-196, 1999.

[DSS08] T.J. Dijkema, Ch. Schwab, and R.P. Stevenson. An adaptive wavelet method for solving high-dimensional elliptic PDEs. Technical report, 2008. To appear in Constr. Approx.

[GK00] M. Griebel and S. Knapek. Optimized tensor-product approximation spaces. Constr. Approx., 16(4):525-540, 2000.

[GO95] M. Griebel and P. Oswald. Tensor product type subspace splittings and multilevel iterative methods for anisotropic problems. Adv. Comput. Math., 4(1-2):171-206, 1995.

[Gri66] P. Grisvard. Commutativité de deux foncteurs d'interpolation et applications. J. Math. Pures Appl. (9), 45:143-206, 1966.

[Gri85] P. Grisvard. Elliptic problems in nonsmooth domains, volume 24 of Monographs and Studies in Mathematics. Pitman (Advanced Publishing Program), Boston, MA, 1985.

[Hoc01] R. Hochmuth. Nonlinear anisotropic boundary value problems - regularity results and multiscale discretizations. Nonlinear Anal., 46(1, Ser. A: Theory Methods):1-18, 2001.

[MP77] V. G. Maz'ya and B. A. Plamenevskii. Elliptic boundary value problems on manifolds with singularities. Probl. Mat. Anal., 6:85-142, 1977.

[MR91] V. G. Maz'ya and J. Rossmann. On the Agmon-Miranda maximum principle for solutions of elliptic equations in polyhedral and polygonal domains. Ann. Global Anal. Geom., 9(3):253-303, 1991.

[Nit05] P.-A. Nitsche. Sparse approximation of singularity functions. Constr. Approx., 21(1):63$81,2005$.

[NW08] E. Novak and H. Woźniakovski. Approximation of infinitely differentiable multivariate functions is intractable. Technical report, 2008. To appear in Journal of Complexity.

[Wer96] A.G. Werschulz. The complexity of the Poisson problem for spaces of bounded mixed derivatives. In The mathematics of numerical analysis (Park City, UT, 1995), volume 32 of Lectures in Appl. Math., pages 895-914. Amer. Math. Soc., Providence, RI, 1996.

[Zen91] Ch. Zenger. Sparse grids. In Parallel algorithms for partial differential equations (Kiel, 1990), volume 31 of Notes Numer. Fluid Mech., pages 241-251. Vieweg, Braunschweig, 1991.

IRMAR, Université de Rennes 1, Campus de Beaulieu, 35042, Rennes Cedex France E-mail address: monique.dauge@univ-rennes1.fr

Korteneg-de Vries Institute for Mathematics, University of Amsterdam, P.O. Box 94248, 1090 GE Amsterdam, The Netherlands

E-mail address: R.P.Stevenson@uva.nl 